\title{
Liouville results for fully nonlinear equations modeled on Hörmander vector fields: I. The Heisenberg group
}

\author{
Martino Bardi ${ }^{1}\left[\right.$ [ $\cdot$ Alessandro Goffi ${ }^{1}$ \\ Received: 11 June 2020 / Revised: 23 October 2020 / Accepted: 9 November 2020 / \\ Published online: 23 December 2020 \\ (c) The Author(s) 2020
}

\begin{abstract}
This paper studies Liouville properties for viscosity sub- and supersolutions of fully nonlinear degenerate elliptic PDEs, under the main assumption that the operator has a family of generalized subunit vector fields that satisfy the Hörmander condition. A general set of sufficient conditions is given such that all subsolutions bounded above are constant; it includes the existence of a supersolution out of a big ball, that explodes at infinity. Therefore for a large class of operators the problem is reduced to finding such a Lyapunov-like function. This is done here for the vector fields that generate the Heisenberg group, giving explicit conditions on the sign and size of the first and zero-th order terms in the equation. The optimality of the conditions is shown via several examples. A sequel of this paper applies the methods to other Carnot groups and to Grushin geometries.
\end{abstract}

Mathematics Subject Classification Primary 35B53 - 35J70 · 35J60; Secondary 49L25 $\cdot 35 \mathrm{H} 20$

Communicated by Y. Giga.

The authors are members of the Gruppo Nazionale per l'Analisi Matematica, la Probabilità e le loro Applicazioni (GNAMPA) of the Istituto Nazionale di Alta Matematica (INdAM). The authors were partially supported by the research project "Nonlinear Partial Differential Equations: Asymptotic Problems and Mean-Field Games" of the Fondazione CaRiPaRo. This research was largely carried out while Alessandro Goffi was Ph.D. fellow at Gran Sasso Science Institute and the main results are part of his Ph.D. thesis.

$\bowtie \quad$ Martino Bardi

bardi@math.unipd.it

Alessandro Goffi

alessandro.goffi@math.unipd.it

1 Department of Mathematics “T. Levi-Civita”, University of Padova, Via Trieste 63, 35121 Padua, Italy 


\section{Contents}

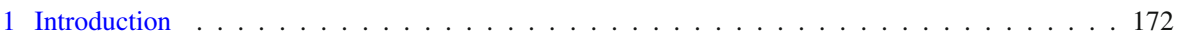

2 A glimpse on the method of proof for linear equations . . . . . . . . . . . . . . . 177

3 The general case . . . . . . . . . . . . . . . . . . . . . . . 178

3.1 An abstract result . . . . . . . . . . . . . . . . . . . . . . 178

3.2 Equations with Hörmander vector fields $\ldots \ldots \ldots \ldots$. . . . . . . . . . . . 181

3.3 Equations driven by Pucci's subelliptic operators . . . . . . . . . . . . . . . . . 183

3.4 Fully nonlinear uniformly subelliptic equations . . . . . . . . . . . . . . . . . . 185

3.5 Normalized $p$-Laplacian . . . . . . . . . . . . . . . . . . . . . . . . . . . 187

4 The Heisenberg vector fields . . . . . . . . . . . . . . . . . . . . . . . . . . 187

4.1 Fully nonlinear PDEs on the Heisenberg group . . . . . . . . . . . . . . 188

4.2 Comparison with the literature and sharpness of the conditions . . . . . . . . . . . 193

4.2 .1 The Euclidean case . . . . . . . . . . . . . . . . . . . . . . . . . . . . . . . . . 194

4.2.2 The Heisenberg case: sublaplacians . . . . . . . . . . . . . . . . . . . . . . . . 195

4.2.3 The Heisenberg case: fully nonlinear operators . . . . . . . . . . . . . . . . . 195

4.3 Equations with Heisenberg Hessian and Euclidean gradient . . . . . . . . . . . . . . . 198

References . . . . . . . . . . . . . . . . . . . . . . . . . . . . . . 199

\section{Introduction}

In this paper we study Liouville properties for viscosity sub- or supersolutions of fully nonlinear degenerate elliptic equations

$$
F\left(x, u, D u, D^{2} u\right)=0 \text { in } \mathbb{R}^{d},
$$

where $F: \mathbb{R}^{d} \times \mathbb{R} \times \mathbb{R}^{d} \times \mathcal{S}_{d} \rightarrow \mathbb{R}, \mathcal{S}_{d}$ being the set of $d \times d$ symmetric matrices, is at least continuous and proper, i.e., nondecreasing in the second entry and non-increasing in the last entry (with respect to the partial order of symmetric matrices). Our main assumption is the existence of a family $\mathcal{X}=\left(X_{1}, \ldots, X_{m}\right)$ of vector fields satisfying the Hörmander bracket generating condition and subunit for $F$ in the following sense: for all $i=1, \ldots, m$

$$
\sup _{\gamma>0} F(x, 0, p, I-\gamma p \otimes p)>0 \quad \forall p \in \mathbb{R}^{d} \text { such that } X_{i}(x) \cdot p \neq 0 .
$$

This generalizes the classical definition of subunit vectors for linear operators by Fefferman and Phong [22] and was introduced in our recent paper [8]. Typical examples are subelliptic equations of the form

$$
G\left(x, u, D u,\left(D_{\mathcal{X}}^{2} u\right)^{*}\right)=0
$$

where $\left(D_{\mathcal{X}}^{2} u\right)_{i j}=X_{i}\left(X_{j} u\right)$ is the intrinsic (or horizontal) Hessian associated to $\mathcal{X}$, $Y^{*}$ is the symmetrized matrix of $Y$, and $G$ is proper and strictly decreasing with respect to the last entry.

Before explaining our results, let us recall some of the many Liouville-type properties for elliptic equations known in the literature, the most related to our work. The classical Liouville theorem for harmonic functions on the whole space states that the only harmonic functions in $\mathbb{R}^{d}$ bounded from above or below are constants, and it is 
a consequence of mean-value formulas or, more generally, of the Harnack inequality. Such result actually holds for classical solutions to more general uniformly elliptic equations, provided the zero-th order coefficient has the appropriate sign for the maximum principle, and the equation is homogeneous, see, e.g., the monograph [26]. For inhomogeneous equations the property is false, for instance $\Delta\left(|x|^{2}\right)=2 d$ in $\mathbb{R}^{d}$.

The Liouville property holds also in the much larger class of merely subharmonic functions (i.e., subsolutions of $-\Delta u=0$ ) bounded from above if the space dimension is $d=2$, by exploiting the behavior of the fundamental solution $\log |x|$ and using the Hadamard Three-Circle Theorem (see, e.g., [38, Theorem 2.29], or Theorem 2.1 below for a different proof). However, this result fails in higher dimensions $d \geq 3$ : for instance, $u_{1}(x):=-\left(1+|x|^{2}\right)^{-1 / 2}$ and $u_{2}(x):=-\left(1+|x|^{2}\right)^{-1}$ are nonpositive subharmonic functions in $\mathbb{R}^{3}$ and, respectively, in $\mathbb{R}^{d}$ with $d \geq 4$.

For linear degenerate elliptic equations, mean-value properties and Harnack-type inequalities were proved in many cases, typically for vector fields $\mathcal{X}$ that generate a stratified Lie group, and Liouville theorems for solutions to such equations were proved, e.g., in [12,17,29,30], see also the references therein.

On the other hand, one does not expect the Liouville property for sub- or supersolutions to $-\Delta \mathcal{X} u=0$ when $\mathcal{X}$ generates a Carnot group, because the intrinsic dimension of this geometry is larger than 2 , which is the maximal one for subharmonic functions.

In fact, in Sect. 4.2 we give simple explicit examples of bounded, non-constant, classical sub- and supersolutions of the sub-Laplace equation in any Heisenberg group $\mathbb{H}^{d}$.

Liouville theorems for nonlinear elliptic equations were first considered by Gidas and Spruck [25] for semilinear equations and then widely investigated, also in the subelliptic and in the quasilinear settings, see, e.g., [2,10,13,14,16,17,40] and the references therein.

For fully nonlinear equations as (1), in the simpler form $F\left(x, D^{2} u\right)=0$ and uniformly elliptic, it was proved in [15, Section 4.3 Remark 4] that continuous viscosity solutions either bounded from above or below are constant. We recall that uniform ellipticity with parameters $\Lambda \geq \lambda>0$ can be defined by means of Pucci's extremal operators (see their definition in Sect. 3.3) as

$$
\mathcal{M}_{\lambda, \Lambda}^{-}(M) \leq F(x, r, p, M)-F(x, r, p, 0) \leq \mathcal{M}_{\lambda, \Lambda}^{+}(M)
$$

for all symmetric matrices $M$. The result is a consequence of the Harnack inequality and comparison with Pucci's operators.

Further related results for solutions of PDEs of the form $F\left(D^{2} u\right)=0$ can be found in [35, Section 1.7] and [4, Theorem 1.7], and in [36, Theorem 1.5] for equations with $F$ depending also on $x$ and $D u$.

The first results for mere sub- or supersolutions of $F\left(x, D^{2} u\right)=0$ are due to Cutrì and Leoni [20]. They proved that if $u \in C\left(\mathbb{R}^{d}\right)$ is either bounded above and satisfying

$$
\mathcal{M}_{\lambda, \Lambda}^{+}\left(D^{2} u\right) \leq 0 \text { in } \mathbb{R}^{d}
$$


in viscosity sense, or bounded below and satisfying

$$
\mathcal{M}_{\lambda, \Lambda}^{-}\left(D^{2} u\right) \geq 0 \text { in } \mathbb{R}^{d}
$$

in viscosity sense, then $u$ is constant provided that $d \leq \frac{\Lambda}{\lambda}+1$. This can be seen as the fully nonlinear analogue of the Liouville theorem for subharmonic functions, since when $\lambda=\Lambda$ one gets the Laplacian (up to constants) and the constraint reads $d \leq 2$. Such conditions are known to be sharp: examples of nontrivial solutions to Pucci's extremal equations when $d>\frac{\Lambda}{\lambda}+1$ can be found in [20, Remark 2] and in Sect. 4.2 below.

This result was extended to the Heisenberg group $\mathbb{H}^{d}$ by Cutrì and Tchou [21, Theorem 5.2] for the inequalities (5) and (6) with $D^{2} u$ replaced by $\left(D_{\mathbb{H} d}^{2} u\right)^{*}$. Here the condition $d \leq \frac{\Lambda}{\lambda}+1$ is replaced by $Q \leq \frac{\Lambda}{\lambda}+1, Q=2 d+2$ standing for the homogeneous dimension of $\mathbb{H}^{d}$. An example of classical subsolution violating the Liouville property when $Q>\frac{\Lambda}{\lambda}+1$ is in Sect. 4.2. This is consistent with the aforementioned failure of Liouville properties for subharmonic functions in the Heisenberg group.

In [20] the authors also prove Liouville results for sub- and supersolutions of $F\left(x, D^{2} u\right)+u^{p}=0$ with $F$ uniformly elliptic, $F(x, 0)=0$ and $p$ in a suitable range. This was recently extended to Carnot groups of Heisenberg type in [27]. See also [3] and [31] for related results.

Liouville properties for PDEs involving gradient terms of the form

$$
F\left(x, D^{2} u\right)+g(|x|)|D u|+h(x) u^{p}=0,
$$

were first investigated by Capuzzo Dolcetta and Cutrì [18]. They assume that $g$ is bounded and such that

$$
-\frac{\Lambda(d-1)}{|x|} \leq g(|x|) \leq \frac{\lambda-\Lambda(d-1)}{|x|}
$$

for $|x|$ large, and use suitable extensions of the Hadamard three-sphere theorem. Note that this is a smallness condition at infinity on the first order terms of the PDE. See also [27] for similar recent results on the Heisenberg group. Related papers for fully nonlinear PDEs with gradient dependence are [36,41] and [19].

A new approach to Liouville properties for sub- and supersolutions of HamiltonJacobi-Bellman elliptic equations involving operators of Ornstein-Uhlenbeck type was introduced in [6], based on the strong maximum principle and the existence of a sort of Lyapunov function for the equation. It was applied in [5] to fully nonlinear uniformly elliptic equations of the form (1) and to some quasilinear hypoelliptic equations, under assumptions on the sign of the coefficients of the first and zero-th order terms, and on their size. Here these terms must be large for large $|x|$, contrary to the results quoted above. In the case of Pucci's operators the results of [5] are different from those in [20] and fit better the treatment of uniformly elliptic equations via the inequalities (4). In Sect. 4.2 we give examples showing their optimality. The paper [33] treats a linear equation on the Heisenberg group in the same spirit. 
In the present paper we study Liouville properties for upper semicontinuous viscosity subsolutions of equations of the form (1) under the condition (2) for a Hörmander family $\mathcal{X}$ and suitable structural conditions for $F$. We further assume the existence of a viscosity supersolution $w$ to (1) outside a compact set such that $\lim _{|x| \rightarrow \infty} w(x)=+\infty$, that we call a Lyapunov function. The bound from above at infinity for $u$ is expressed by

$$
\limsup _{|x| \rightarrow \infty} \frac{u(x)}{w(x)} \leq 0
$$

In this case we call Liouville property for subsolutions of (1) the following:

$$
\text { if } u \in \mathrm{USC}\left(\mathbb{R}^{d}\right) \text { is a viscosity subsolution to (1) satisfying (7) }
$$

for a Lyapunov function $w$, then $u$ is constant.

Symmetrically, a Liouville property for LSC supersolutions $v$ can be deduced assuming the existence of a viscosity subsolution $W$ for Eq. (1) outside a compact set such that $\lim _{|x| \rightarrow \infty} W(x)=-\infty$, that we call a negative Lyapunov function. The bound from below at infinity for $v$ is

$$
\limsup _{|x| \rightarrow \infty} \frac{v(x)}{W(x)} \leq 0
$$

Now we call Liouville property for supersolutions of (1) the following:

$$
\begin{array}{r}
\text { if } v \in \operatorname{LSC}\left(\mathbb{R}^{d}\right) \text { is a viscosity supersolution to (1) satisfying (8) } \\
\text { for a negative Lyapunov function } W \text {, then } v \text { is constant. }
\end{array}
$$

Our main motivation are equations of the form (3), uniformly subelliptic in the sense that $G$ satisfies the inequalities (4) with Pucci operators $\mathcal{M}_{\lambda, \Lambda}^{ \pm}$acting on $m$ dimensional instead of $d$-dimensional symmetric matrices. In the first part we prove a general result assuming in addition a natural subadditivity condition on $F$ for subsolutions and a symmetric superadditivity condition for supersolutions (resp., (S1) and (S2) of Sect. 3.1), which for linear equations correspond to the homogeneity.

Here we adapt the approach of [5] to degenerate equations by means of the new strong maximum and minimum principles obtained by the authors in the recent paper [8] using the generalized subunit vectors for fully nonlinear equations (2).

In the second part of the paper we find more explicit sufficient conditions for the Liouville properties in the case of the Heisenberg group $\mathbb{H}^{d}$ by taking $w=\log \rho$ as Lyapunov function, where $\rho$ is a norm 1-homogeneous with respect to the dilations of the group $\mathbb{H}^{d}$. As must be expected from the results quoted before, these assumptions concern the sign and the strength of either the first or the zero-th order terms in the equation (or both). They are related to recurrence conditions in the probabilistic literature, and are a form of dissipativity (cfr., e.g., [37]). An example of our results, 
for the uniformly subelliptic equation

$$
G\left(x, u, D_{\mathbb{H}^{d}} u,\left(D_{\mathbb{H}^{d}}^{2} u\right)^{*}\right)=0, \quad \text { in } \mathbb{R}^{2 d+1},
$$

where $D_{\mathbb{H}} u$ and $D_{\mathbb{H} d}^{2} u$ are the horizontal gradient and Hessian in $\mathbb{H}^{d}$, is the following: if

$$
G(x, r, p, X) \geq \mathcal{M}_{\lambda, \Lambda}^{-}(X)+\inf _{\alpha \in A}\left\{c^{\alpha}(x) r-b^{\alpha}(x) \cdot p\right\}
$$

with $c^{\alpha} \geq 0$, we prove the Liouville property for subsolutions under the condition

$$
\sup _{\alpha \in A}\left\{b^{\alpha}(x) \cdot \frac{\eta}{\left|x_{H}\right|^{2}}-c^{\alpha}(x) \frac{\rho^{4}}{\left|x_{H}\right|^{2}} \log \rho\right\} \leq \lambda-\Lambda(Q-1) \quad \text { for }|x| \geq R \text {, }
$$

where $x_{H}:=\left(x_{1}, \ldots, x_{2 d}\right) \neq 0_{\mathbb{R}^{2 d}}, \eta \in \mathbb{R}^{2 d}$ is defined by $\eta_{i}=x_{i}\left|x_{H}\right|^{2}+x_{i+d} x_{2 d+1}$, $\eta_{i+d}=x_{i}\left|x_{H}\right|^{2}-x_{i} x_{2 d+1}$, for $i=1, \ldots, d$, and $Q=2 d+2$ is the homogeneous dimension of $\mathbb{H}^{d}$. This condition is satisfied if either $c^{\alpha}>0$ or $b^{\alpha}(x) \cdot \eta<0$ for $x$ large, and under suitable growth conditions at infinity of the data. Here we exploit the analysis of $D_{\mathbb{H} d}^{2} \rho$ in the Heisenberg group done in [21]. In Sect. 4.2 we use again the norm $\rho$ to discuss the sharpness of this condition.

In our companion paper [7] we apply the general results of Sect. 3 to other classical families of Hörmander vector fields, namely, the generators of H-type groups, free step 2 Carnot groups, and Grushin-type fields, whose associated geometry has not a group structure. In those geometries there is still a homogeneous norm $\rho$ with respect to suitable dilations, and we make in [7] a new careful analysis of $D_{\mathbb{H} d}^{2} \rho$ to get Liouville results in those contexts.

It is well known that Liouville properties have many applications to various issues. We are motivated, in particular, by their consequences in ergodic problems, large time stabilization in parabolic equations, and singular perturbation problems, as in, e.g., $[5,6,33,34]$. For other forms of Liouville-type theorems for different equations let us also mention [9] in the Heisenberg group, the recent paper [32] for PDEs arising in conformal geometry, and [11] for versions of Pucci's extremal equations with different degeneracies than in our work.

The paper is organised as follows. In Sect. 2 we explain the approach to Liouville properties based on Lyapunov functions and strong maximum principles in the simple case of classical subsolutions of linear equations, for the reader's convenience, and discuss some related literature. Section 3 presents an abstract result and its various applications to nonlinear equations with general Hörmander vector fields. In Sect. 4 we study PDEs involving the generators of the Heisenberg group $\mathbb{H}^{d}$, in the form (9) where only the horizontal gradient appears, as well as in the form (3) involving the Euclidean gradient. Section 4.2 makes a detailed comparison with the literature, in the cases of $\mathbb{R}^{d}$ and $\mathbb{H}^{d}$, and discusses by means of explicit examples the optimality of the sufficient conditions for Liouville properties. 


\section{A glimpse on the method of proof for linear equations}

Before showing our main results, we present the proof of a Liouville-type theorem for classical $C^{2}$ subsolutions to linear uniformly elliptic equations in the Euclidean framework, which serves as a guideline for our proof in the nonlinear and subelliptic setting. It uses only classical arguments such as strong maximum and comparison principles, but not Harnack inequalities.

Theorem 2.1 Assume the operator $L u:=-\operatorname{Tr}\left(a(x) D^{2} u\right)+b(x) \cdot D u+c(x) u$ is uniformly elliptic, with $a: \mathbb{R}^{d} \rightarrow \mathcal{S}_{d}, b: \mathbb{R}^{d} \rightarrow \mathbb{R}^{d}, c: \mathbb{R}^{d} \rightarrow[0,+\infty)$ locally bounded. Suppose there exists $w \in C^{2}\left(\mathbb{R}^{d} \backslash\{0\}\right)$ such that, for some $R>0$,

(i) $L w \geq 0$ for $|x|>R$

(ii) $\lim _{|x| \rightarrow+\infty} w=+\infty$.

Let also $u \in C^{2}\left(\mathbb{R}^{d}\right)$ be such that $L u \leq 0$ and $0 \leq u(x) \leq C$ in $\mathbb{R}^{d}$. Then $u$ is constant.

Remark 2.2 This result is essentially a special case, e.g., of [5, Theorem 2.1] and applies to the case of the Laplacian (i.e. $a_{i j}=\delta_{i j}$ and $b=0$ ) when $d \leq 2$; therefore it gives a different proof of the Liouville theorem for subsolutions, see e.g. [38, Theorem 2.29]. Indeed, the function $w:=\log |x|$ fulfills the above assumptions, giving thus that every subharmonic function bounded from above is constant. However, as pointed out in the introduction, this is not the case when $d \geq 3$, where $w$ is no longer a classical supersolution of $-\Delta u=0$. A more general result in the context of Riemannian manifolds can be found in [28, Corollary 7.7] under the same sufficient conditions (i)-(ii). It applies to subsolutions of $-\Delta u+b(x) \cdot D u=0$ in any space dimension $d$ under assumptions on the drift $b$ implying the existence of a Lyapunov-like function $w$ (cfr. [5]).

Proof For $\zeta>0$ set

$$
v_{\zeta}(x):=u(x)-\zeta w(x) \text { for }|x| \geq \bar{R}
$$

for some $\bar{R}>R>0$. Clearly, $v_{\zeta} \in C^{2}\left(\Omega_{\bar{R}}\right)$, where $\Omega_{\bar{R}}:=\left\{x \in \mathbb{R}^{d}:|x| \geq \bar{R}\right\}$, and

$$
L v_{\zeta}=L u-\zeta L w \leq 0 \text { for every } x \text { such that }|x|>\bar{R} \text {. }
$$

Define $C_{\zeta}:=\max _{\{|x|=\bar{R}\}} v_{\zeta}(x)$. Since

$$
\lim _{|x| \rightarrow+\infty} v_{\zeta}(x)=-\infty
$$

there exists $K_{\zeta}>\bar{R}$ such that $v_{\zeta}<C_{\zeta}$ for every $x$ such that $|x| \geq K_{\zeta}$. By the weak maximum principle (see [26, Corollary 3.2]) on the set $\left\{x \in \mathbb{R}^{d}: \bar{R}<|x|<K_{\zeta}\right\}$ we have

$$
\max _{\left\{x \in \mathbb{R}^{d}: \bar{R}<|x|<K_{\zeta}\right\}} v_{\zeta}(x)=\max _{\left\{x \in \mathbb{R}^{d}:|x|=\bar{R} \text { or }|x|=K_{\zeta}\right\}} v_{\zeta}(x)=C_{\zeta} .
$$


Since $v_{\zeta}(x)<C_{\zeta}$ for every $x$ such that $|x| \geq K_{\zeta}$, we get, for all $|y| \geq \bar{R}$,

$$
v_{\zeta}(y) \leq C_{\zeta} \leq \max _{\left\{x \in \mathbb{R}^{d}:|x|=\bar{R}\right\}} u-\zeta \min _{\left\{x \in \mathbb{R}^{d}:|x|=\bar{R}\right\}} w .
$$

On one hand, letting $\zeta \rightarrow 0$ we conclude

$$
u(y) \leq \max _{\left\{x \in \mathbb{R}^{d}:|x|=\bar{R}\right\}} u, \quad \text { for all }|y| \geq \bar{R}
$$

On the other hand, owing to the weak maximum principle in the set $B(0, \bar{R})$ we obtain

$$
u(y) \leq \max _{\left\{x \in \mathbb{R}^{d}:|x|=\bar{R}\right\}} u, \quad \text { for all }|y|<\bar{R}
$$

Combining the above inequalities one concludes

$$
u(y) \leq \max _{\left\{x \in \mathbb{R}^{d}:|x|=\bar{R}\right\}} u, \quad \text { for all } y \in \mathbb{R}^{d} .
$$

Hence, $u$ attains its nonnegative maximum at some point of $\partial B(0, \bar{R})$ and then the conclusion follows by the strong maximum principle for classical linear uniformly elliptic equations [26, Theorem 3.5].

Remark 2.3 The same result remains true if $L$ is replaced by a degenerate elliptic operator $L_{\mathcal{X}} u:=-\sum_{i, j} X_{i} X_{j} u+b(x) \cdot D_{\mathcal{X}} u+c(x) u$, provided the vector fields $\mathcal{X}$ satisfy the Hörmander condition and $b: \mathbb{R}^{d} \rightarrow \mathbb{R}^{m}, m \leq d$, is smooth, the proof being exactly the same thanks to Bony strong maximum principle for subelliptic equations. An example of such result is [33, Proposition 3.1].

Note also that the assumption $u \leq C$ can be replaced by $\lim \sup _{|x| \rightarrow \infty} u(x) / w(x) \leq$ 0 , whereas the sign condition $u \geq 0$ can be dropped if $c \equiv 0$.

We further remark that, when $b \equiv 0, L$ reduces to a Schrödinger-type operator. When $u$ is a solution of the equation $-\Delta u+c u=0$, the Liouville property is proved in [28, Corollary 13.7] under the same sufficient conditions (i)-(ii) on Riemannian manifolds, and it is connected to recurrence and non-explosive properties of Brownian motions on Riemannian manifolds, see [28, Theorem 5.1 and Section 13.2]. Our result is more general in that it allows $u$ to be merely a subsolution to the equation.

We also refer to [37] for a control theoretic interpretation of the Liouville property for Ornstein-Uhlenbeck operators.

\section{The general case}

\subsection{An abstract result}

In this section we consider a general equation of the form

$$
F\left(x, u, D u, D^{2} u\right)=0 \text { in } \mathbb{R}^{d} .
$$


We will denote $F[u]:=F\left(x, u, D u, D^{2} u\right)$ and make the following assumptions

(i) $F: \mathbb{R}^{d} \times \mathbb{R} \times \mathbb{R}^{d} \times \mathcal{S}_{d} \rightarrow \mathbb{R}$ is continuous, proper, satisfies

$$
F[\varphi-\psi] \leq F[\varphi]-F[\psi] \text { for all } \varphi, \psi \in C^{2}\left(\mathbb{R}^{d}\right)
$$

and $F(x, r, 0,0) \geq 0$ for every $x \in \Omega$ and $r \geq 0$.

(ii) $F$ satisfies the comparison principle in any bounded open set $\Omega$, namely, if $u$ and $v$ are, respectively, a viscosity sub- and supersolution of (10) such that $u \leq v$ on $\partial \Omega$, then $u \leq v$ in $\Omega$.

(iii) There exists $R_{o} \geq 0$ and $w \in \operatorname{LSC}\left(\mathbb{R}^{d}\right)$ viscosity supersolution of (10) for $|x|>R_{o}$ and satisfying $\lim _{|x| \rightarrow \infty} w(x)=+\infty$.

(iv) $F$ satisfies the strong maximum principle, namely, any viscosity subsolution of (10) that attains an non-negative maximum must be constant.

To prove the analogous results for viscosity supersolutions we need to replace (i) and (iii)-(iv) above by

(i') $F$ is continuous, proper, satisfies

$$
F[\varphi-\psi] \geq F[\varphi]-F[\psi] \text { for all } \varphi, \psi \in C^{2}\left(\mathbb{R}^{d}\right)
$$

and $F(x, r, 0,0) \leq 0$ for every $x \in \Omega$ and $r \leq 0$.

(iii') There exists $R_{o} \geq 0$ and $W \in \operatorname{USC}\left(\mathbb{R}^{d}\right)$ viscosity subsolution to (10) for $|x|>$ $R_{o}$ and satisfying $\lim _{|x| \rightarrow \infty} W(x)=-\infty$.

$\left(\right.$ iv $\left.^{\prime}\right) F$ satisfies the strong minimum principle.

The next result extends the proof of Theorem 2.1 for linear equations to the fully nonlinear degenerate setting. Its proof is essentially the same as the one done in [5] for HJB equations, so we only outline it for the reader's convenience.

Proposition 3.1 Assume (i)-(iv). If $u \geq 0$, then (LP1) holds for (10).

Proof Define $u_{\zeta}(x):=u(x)-\zeta w(x)$ for $\zeta>0$. Possibly increasing $R_{o}$, we can assume that $u$ is not constant in $\bar{B}\left(0, R_{o}\right):=\left\{x \in \mathbb{R}^{m}:|x| \leq R_{0}\right\}$, otherwise we are done. Set

$$
C_{\zeta}:=\max _{|x| \leq R_{o}} u_{\zeta}(x)
$$

Note that $F\left[C_{\zeta}\right] \geq 0$ and $C_{\zeta}>0$ for all $\zeta$ sufficiently small. In fact, if $C_{\zeta}=0$, by letting $\zeta \rightarrow 0$ we get $u(x)=0$ for every $x$ with $|x| \leq R_{o}$, a contradiction with $u$ not constant in $\bar{B}\left(0, R_{o}\right)$.

The growth condition (7) implies

$$
\limsup _{|x| \rightarrow \infty} \frac{u_{\zeta}(x)}{w(x)} \leq-\zeta<0 \quad \forall \zeta>0 .
$$

As a consequence, we have

$$
\lim _{|x| \rightarrow+\infty} u_{\zeta}(x)=-\infty
$$


Then, for all $\zeta>0$ there exists $R_{\zeta}>R_{O}$ such that

$$
u_{\zeta}(x) \leq C_{\zeta} \quad \text { for all }|x| \geq R_{\zeta} .
$$

The main step is proving that $u_{\zeta}$ is a viscosity subsolution of $F[u]=0$ in $\{x \in$ $\left.\mathbb{R}^{d}:|x|>R_{o}\right\}$. Take $\bar{x}$ and $\varphi$ smooth such that $0=\left(u_{\zeta}-\varphi\right)(\bar{x})>\left(u_{\zeta}-\varphi\right)(x)$ for all $x$. Assume by contradiction that $F[\varphi(\bar{x})]>0$. Then for some $\delta>0$ and $0<r<|\bar{x}|-R_{O}$

$$
F[\varphi-\delta]>0 \text { in } B(\bar{x}, r) .
$$

Next take $0<k<\delta$ such that $u_{\zeta}-\varphi \leq-k<0$ on $\partial B(\bar{x}, r)$. We claim that $\zeta w+\varphi-k$ satisfies $F[\zeta w+\varphi-k] \geq 0$ in $B(\bar{x}, r)$. Indeed, take $\tilde{x} \in B(\bar{x}, r)$ and $\psi$ smooth such that $\zeta w+\varphi-k-\psi$ has a minimum at $\tilde{x}$. Using that $w$ is a viscosity supersolution to (10), $F$ proper, (S1), and (14) we get

$$
\begin{aligned}
0 \leq F[\psi(\tilde{x})-\varphi(\tilde{x})+k] & \leq F[\psi(\tilde{x})-\varphi(\tilde{x})+\delta] \\
& \leq F[\psi(\tilde{x})]-F[\varphi(\tilde{x})-\delta]<F[\psi(\tilde{x})] .
\end{aligned}
$$

Then $\zeta w+\varphi-k$ is a supersolution to $F[u]=0$ in $B(\bar{x}, r)$ and $u \leq \zeta w+\varphi-k$ on $\partial B(\bar{x}, r)$, so the comparison principle gives $u \leq \zeta w+\varphi-k$ in $B(\bar{x}, r)$, in contradiction with the fact that $u(\bar{x})=\zeta w(\bar{x})+\varphi(\bar{x})$.

Now we can use the comparison principle in $\Omega=\left\{x: R_{O}<|x|<R_{\zeta}\right\}$ and (13) to get $u_{\zeta} \leq C_{\zeta}$ in $\Omega$. Therefore we have

$$
u_{\zeta}(x) \leq C_{\zeta} \quad \text { for all }|x| \geq R_{o}
$$

By letting $\zeta \rightarrow 0^{+}$we obtain

$$
u(x) \leq \max _{|y| \leq R_{o}} u(y) \text { for all } x \in \mathbb{R}^{d},
$$

and hence $u$ attains its maximum on $\mathbb{R}^{d}$ at some point. Since $u \geq 0$ the strong maximum principle gives the desired conclusion.

Remark 3.2 Note that if $u$ is bounded above, then (7) is satisfied.

The next result says that the assumption $u \geq 0$ can be dropped provided $r \mapsto$ $F(x, r, p, X)$ is constant: this will be the case for some HJB operators we discuss in the next sections.

Corollary 3.3 Assume (i)-(iv). Assume $r \mapsto F(x, r, p, X)$ is constant for all $x, p, X$ and $F(x, r, 0,0)=0$ for every $x \in \Omega$. Then (LP1) holds for (10).

Proof The proof goes along the same lines as Proposition 3.1. It is sufficient to note that since $r \mapsto F(x, r, p, X)$ is constant for all $x, p, X, u+|u(\bar{x})|, \bar{x}$ standing for the maximum point in Proposition 3.1, is again a subsolution, and one concludes.

A symmetric result holds for the case of supersolutions to (10), see [5] for the details of the proof. 
Proposition 3.4 Assume ( $\left.i^{\prime}\right)$, (ii), (iii') and (iv'). Then (LP2) holds for (10) provided that either $v \leq 0$, or $r \mapsto F(x, r, p, X)$ is constant for all $x, p, X$ and $F(x, r, 0,0)=$ 0 for every $x \in \Omega$.

\subsection{Equations with Hörmander vector fields}

In this section we discuss Liouville properties for PDEs over Hörmander vector fields. We recall that the vector fields $Z_{1}, \ldots, Z_{m}$ satisfy the Hörmander's rank condition if (H) the vector fields are smooth and the Lie algebra generated by them has full rank $d$ at each point.

The classical smoothness requirement on $Z_{i}$ is $C^{\infty}$, but it can be reduced to $C^{k}$ for a suitable $k$, and considerably more if the Lie brackets are interpreted in a generalized sense, as, e.g., in [23], see Remark 3.8.

Before stating the main result for subsolutions, we recall a crucial scaling assumption for the validity of the strong maximum principle for fully nonlinear subelliptic equations together with the concept of generalized subunit vector field.

(SC) For some $\phi:(0,1] \rightarrow(0,+\infty], F$ satisfies

$$
F(x, \xi s, \xi p, \xi X) \geq \phi(\xi) F(x, s, p, X)
$$

for all $\xi \in(0,1], s \in[-1,0], x \in \Omega, p \in \mathbb{R}^{d} \backslash\{0\}$, and $X \in \mathcal{S}_{d}$;

Definition $3.5 Z \in \mathbb{R}^{d}$ is a generalized subunit vector (briefly, SV) for $F=$ $F(x, r, p, X)$ at $x \in \Omega$ if

$$
\sup _{\gamma>0} F(x, 0, p, I-\gamma p \otimes p)>0 \quad \forall p \in \mathbb{R}^{d} \text { such that } Z \cdot p \neq 0 ;
$$

$Z: \Omega \rightarrow \mathbb{R}^{d}$ is a subunit vector field (briefly, SVF) if $Z(x)$ is SV for $F$ at $x$ for every $x \in \Omega$.

The name is motivated by the notion introduced by Fefferman and Phong [22] for linear operators. The following strong maximum principle has been proved in [8] and asserts the propagation of maxima for viscosity subsolutions to $F=0$ along the trajectories of generalized subunit vector fields.

Theorem 3.6 Assume that F satisfies (i), (SC), and it has a locally Lipschitz subunit vector field Z. Suppose that $u \in \operatorname{USC}(\Omega)$ is a viscosity subsolution to (10) attaining a nonnegative maximum at $x_{0} \in \Omega$. Then, $u(x)=u\left(x_{0}\right)=\max _{\Omega} u$ for all $x=y(s)$ for some $s \in \mathbb{R}$, where $y^{\prime}(t)=Z(y(t))$ and $y(0)=x_{0}$.

More generally, the strong maximum principle holds when $F$ has a family of subunit vector fields $Z_{i}, i=1, \ldots, m$, by considering the control system

$$
y^{\prime}(t)=\sum_{i=1}^{m} Z_{i}(y(t)) \beta_{i}(t),
$$


where $\beta_{i}$ are measurable functions taking values in a fixed neighborhood of the origin. If this system has the so-called property of bounded time controllability, i.e.

$$
\begin{aligned}
& \forall x_{0}, x_{1} \in \Omega \quad \exists \text { a trajectory } y(\cdot) \text { of }(15) \text { with } y(0)=x_{0}, \\
& y(s)=x_{1}, y(t) \in \Omega \forall t \in[0, s],
\end{aligned}
$$

then the maximum propagates to the whole $\Omega$. A sufficient condition implying the above controllability condition is the full rank condition $(\mathrm{H})$.

Theorem 3.7 Let $F$ be such that (i), (ii), (iii), and (SC) hold. Furthermore assume that $F$ admits $Z_{1}, \ldots, Z_{m}$ generalized subunit vector fields satisfying the Hörmander condition (H). Then (LP1) holds for (10) if either $u \geq 0$, or $r \mapsto F(x, r, p, X)$ is constant for all $x, p, X$ and $F(x, r, 0,0)=0$ for every $x \in \Omega$.

Remark 3.8 In view of the above discussion, the assumption $(\mathrm{H})$ on the vector fields $Z_{i}$ can be weakened to $C^{1,1}$ regularity plus the controllability property (BTC) for the control system (15). However, note that the Hörmander condition is satisfied in the context of Carnot groups and Grushin geometries, which are the main cases treated in this manuscript and the forthcoming paper [7].

Proof The proof is a consequence of Proposition 3.1, Corollary 3.3, and the strong maximum principle Theorem 3.6, or Corollary 2.6 in [8].

Similarly, in the case of supersolutions we have the following result by replacing (SC) with

$\left(\mathrm{SC}^{\prime}\right)$ For some $\phi:(0,1] \rightarrow(0,+\infty], F$ satisfies

$$
F(x, \xi s, \xi p, \xi X) \leq \phi(\xi) F(x, s, p, X)
$$

for all $\xi \in(0,1], s \in[0,1], x \in \Omega, p \in \mathbb{R}^{d} \backslash\{0\}$, and $X \in \mathcal{S}_{d} ;$

and the condition in Definition 3.5 for being a subunit vector is replaced with

$$
\inf _{\gamma>0} F(x, 0, p, \gamma p \otimes p-I)<0 \quad \forall p \in \mathbb{R}^{d} \text { such that } Z \cdot p \neq 0 \text {. }
$$

The proof is a simple consequence of Proposition 3.4, and the strong minimum principle Corollary 2.12 in [8].

Theorem 3.9 Let $F$ be such that (i'), (ii), (iii'), and (SC') hold. Furthermore, assume that $F$ admits $Z_{1}, \ldots, Z_{m}$ generalized subunit vector fields satisfying the Hörmander condition (H). Then (LP2) holds for (10) if either $v \leq 0$, or $r \mapsto F(x, r, p, X)$ is constant for all $x, p, X$ and $F(x, r, 0,0)=0$ for every $x \in \Omega$.

Next we apply the last two theorems to subelliptic equations of the form

$$
G\left(x, u, D_{\mathcal{X}} u,\left(D_{\mathcal{X}}^{2} u\right)^{*}\right)=0, \quad \text { in } \mathbb{R}^{d},
$$


where $\mathcal{X}=\left(X_{1}, \ldots, X_{m}\right)$ are $C^{1,1}$ vector fields on $\mathbb{R}^{d}$ satisfying the Hörmander condition (H), $D_{\mathcal{X}} u:=\left(X_{1} u, \ldots, X_{m} u\right),\left(D_{\mathcal{X}}^{2} u\right)_{i j}:=X_{i}\left(X_{j} u\right)$, and $Y^{*}$ is the symmetrized matrix of $Y$. Here $G: \mathbb{R}^{d} \times \mathbb{R} \times \mathbb{R}^{m} \times \mathcal{S}_{m} \rightarrow \mathbb{R}$ is at least continuous, proper, satisfying (S1), and it is elliptic for any $x$ and $p$ fixed in the following sense:

$$
\sup _{\gamma>0} G(x, 0, q, X-\gamma q \otimes q)>0 \quad \forall x \in \Omega, q \in \mathbb{R}^{m}, q \neq 0, X \in \mathcal{S}_{m} .
$$

After choosing a basis in Euclidean space we write $X_{j}=\sigma^{j} \cdot D$, with $\sigma^{j}: \mathbb{R}^{d} \rightarrow$ $\mathbb{R}^{d}$, and $\sigma=\sigma(x)=\left[\sigma^{1}(x), \ldots, \sigma^{m}(x)\right] \in \mathbb{R}^{d \times m}$. Then

$$
D_{\mathcal{X}} u=\sigma^{T} D u=\left(\sigma^{1} \cdot D u, \ldots, \sigma^{m} \cdot D u\right)
$$

and

$$
X_{i}\left(X_{j} u\right)=\left(\sigma^{T} D^{2} u \sigma\right)_{i j}+\left(D \sigma^{j} \sigma^{i}\right) \cdot D u
$$

Therefore, for $u \in C^{2}$,

$\left(D_{\mathcal{X}}^{2} u\right)^{*}=\sigma^{T} D^{2} u \sigma+g(x, D u), \quad(g(x, P))_{i j}:=\frac{1}{2}\left[\left(D \sigma^{j} \sigma^{i}\right) \cdot p+\left(D \sigma^{i} \sigma^{j}\right) \cdot p\right]$,

and we can rewrite the Eq. (16) in Euclidean coordinates, i.e., in the form $F\left(x, u, D u, D^{2} u\right)=0$, by taking

$$
F(x, r, p, X)=G\left(x, r, \sigma^{T}(x) p, \sigma^{T}(x) X \sigma(x)+g(x, D u)\right) .
$$

The ellipticity condition (17) implies that the vector fields $X_{j}=\sigma^{j} \cdot D$ are subunit for $F$ (cfr. [8, Lemma 3.1]). Moreover, if $G$ satisfies (SC) or $\left(\mathrm{SC}^{\prime}\right)$, also $F$ does. Assume finally that $F$ given by (18) satisfies property (ii) about the weak comparison principle. Then we have the following.

Corollary 3.10 Under the assumptions listed above the Eq. (16) has the Liouville property for viscosity subsolutions of Theorem 3.7.

\subsection{Equations driven by Pucci's subelliptic operators}

Given a family of $m$ vector fields and the corresponding Hessian matrix, we consider the Pucci's extremal operators over such matrices instead of the classical Euclidean Hessians. Following Caffarelli and Cabré [15], we fix $0<\lambda \leq \Lambda$, denote with $\mathcal{S}_{m}$ the set of $m \times m$ symmetric matrices, and let

$$
\mathcal{A}_{\lambda, \Lambda}:=\left\{A \in \mathcal{S}_{m}: \lambda|\xi|^{2} \leq A \xi \cdot \xi \leq \Lambda|\xi|^{2}, \forall \xi \in \mathbb{R}^{m}\right\}
$$

For $M \in \mathcal{S}_{m}$ the maximal and minimal operator are defined as

$$
\mathcal{M}_{\lambda, \Lambda}^{+}(M):=\sup _{A \in \mathcal{A}_{\lambda, \Lambda}} \operatorname{Tr}(-A M), \quad \mathcal{M}_{\lambda, \Lambda}^{-}(M):=\inf _{A \in \mathcal{A}_{\lambda, \Lambda}} \operatorname{Tr}(-A M) .
$$


If $e_{1} \leq \cdots \leq e_{d}$ are the ordered eigenvalues of the matrix $M$, one can check that $[15$, Section 2.2]

$$
\mathcal{M}_{\lambda, \Lambda}^{+}(M)=-\Lambda \sum_{e_{k}<0} e_{k}-\lambda \sum_{e_{k}>0} e_{k}, \quad \mathcal{M}_{\lambda, \Lambda}^{-}(M)=-\Lambda \sum_{e_{k}>0} e_{k}-\lambda \sum_{e_{k}<0} e_{k}
$$

Now we prove the Liouville property for subsolutions of the equation

$$
\mathcal{M}_{\lambda, \Lambda}^{-}\left(\left(D_{\mathcal{X}}^{2} u\right)^{*}\right)+H_{i}\left(x, u, D_{\mathcal{X}} u\right)=0 \text { in } \mathbb{R}^{d},
$$

where

$$
H_{i}(x, r, p):=\inf _{\alpha \in A}\left\{c^{\alpha}(x) r-b^{\alpha}(x) \cdot p\right\}
$$

and for supersolutions of

$$
\mathcal{M}_{\lambda, \Lambda}^{+}\left(\left(D_{\mathcal{X}}^{2} u\right)^{*}\right)+H_{S}\left(x, u, D_{\mathcal{X}} u\right)=0 \text { in } \mathbb{R}^{d}
$$

where

$$
H_{S}(x, r, p):=\sup _{\alpha \in A}\left\{c^{\alpha}(x) r-b^{\alpha}(x) \cdot p\right\} .
$$

Here $A$ is a set of indices such that $H_{i}$ and $H_{s}$ are finite. We assume that $b^{\alpha}: \mathbb{R}^{d} \rightarrow \mathbb{R}^{m}$ is locally Lipschitz in $x$ uniformly in $\alpha$, i.e., for all $R>0$ there exists $K_{R}>0$ such that

$$
\sup _{|x|,|y| \leq R, \alpha \in A}\left|b^{\alpha}(x)-b^{\alpha}(y)\right| \leq K_{R}|x-y|
$$

and

$$
c^{\alpha}(x) \geq 0 \text { and continuous in }|x| \leq R \text { uniformly in } \alpha .
$$

Corollary 3.11 Assume the vector fields $\mathcal{X}$ are $C^{1,1}$ and satisfy the Hörmander condition $(H)$.

(a) Under the previous assumptions on $H_{i}$, (LP1) holds for (20) provided that either $u \geq 0$ or $c^{\alpha}(x) \equiv 0$.

(b) Under the previous assumptions on $H_{s}$, (LP2) holds for (22) provided that either $v \leq 0$ or $c^{\alpha}(x) \equiv 0$.

Proof The proofs of (a) and (b) are consequences, respectively, of Theorems 3.7 and 3.9. The operators $\mathcal{M}_{\lambda, \Lambda}^{-}\left(\left(D_{\mathcal{X}}^{2} u\right)^{*}\right)$ and $\mathcal{M}_{\lambda, \Lambda}^{+}\left(\left(D_{\mathcal{X}}^{2} u\right)^{*}\right)$ enjoy, respectively, the property (S1) and (S2) as a consequence of the property of duality (i.e., $\mathcal{M}_{\lambda, \Lambda}^{-}(M)=$ $\left.-\mathcal{M}_{\lambda, \Lambda}^{+}(-M)\right)$ and the inequalities

$$
\begin{aligned}
& \mathcal{M}_{\lambda, \Lambda}^{-}(M+N) \leq \mathcal{M}_{\lambda, \Lambda}^{-}(M)+\mathcal{M}_{\lambda, \Lambda}^{+}(N), \\
& \mathcal{M}_{\lambda, \Lambda}^{+}(M+N) \geq \mathcal{M}_{\lambda, \Lambda}^{-}(M)+\mathcal{M}_{\lambda, \Lambda}^{+}(N)
\end{aligned}
$$


for every $M, N \in \mathcal{S}_{d}$, see [15, Lemma 2.10 ]. Moreover, they are positively 1homogeneous, so they satisfy the scalings (SC) and (SC').

The comparison principle (ii) holds for both equations in view of [8, Example 4.6]. Finally, observe that when $c^{\alpha} \equiv 0$, then $G(x, r, 0,0)=0$ for every $x \in \Omega, r \in \mathbb{R}$, and $r \mapsto G(x, r, p, X)$ is constant for every $x, p, X$.

Corollary 3.11 concerns only operators that are either convex or concave with respect to the derivatives of the solution. However, we will use it in the next Sect. 3.4 to study general fully nonlinear uniformly subelliptic equations.

A different, although similar, class of extremal operators was introduced by Pucci in the seminal paper [39] (for the Euclidean Hessian). Here we consider them on the Hessian associated to the vector fields $\mathcal{X}$. Consider for $\alpha>0$ the class of matrices

$$
\mathcal{B}_{\alpha}:=\left\{A \in \mathcal{S}_{m}: A \xi \cdot \xi \geq \alpha|\xi|^{2}, \operatorname{Tr}(A)=1, \forall \xi \in \mathbb{R}^{m}\right\}
$$

and define

$$
\mathcal{P}_{\alpha}^{+}(M):=\sup _{A \in \mathcal{B}_{\alpha}} \operatorname{Tr}(-A M), \quad \mathcal{P}_{\alpha}^{-}(M)=\inf _{A \in \mathcal{B}_{\alpha}} \operatorname{Tr}(-A M)
$$

As pointed out in [39] (see also [26, Chapter 17]), these operators can be rewritten in terms of the ordered eigenvalues of the matrix $M \in \mathcal{S}_{m}$ as follows

$$
\begin{aligned}
& \mathcal{P}_{\alpha}^{+}(M)=-\alpha \sum_{k=2}^{m} e_{k}-[1-(m-1) \alpha] e_{1}=-\alpha \operatorname{Tr}(M)-(1-m \alpha) e_{1} \\
& \mathcal{P}_{\alpha}^{-}(M)=-\alpha \sum_{k=1}^{m-1} e_{k}-[1-(m-1) \alpha] e_{m}=-\alpha \operatorname{Tr}(M)-(1-m \alpha) e_{m}
\end{aligned}
$$

The Liouville properties of Corollary 3.11 hold under the same assumptions for the Eqs. (20) and (22) with the operators $\mathcal{M}_{\lambda, \Lambda}^{-}\left(\left(D_{\mathcal{X}}^{2} u\right)^{*}\right)$ and $\mathcal{M}_{\lambda, \Lambda}^{+}\left(\left(D_{\mathcal{X}}^{2} u\right)^{*}\right)$ replaced, respectively, by $\mathcal{P}_{\alpha}^{-}\left(\left(D_{\mathcal{X}}^{2} u\right)^{*}\right)$ and $\mathcal{P}_{\alpha}^{+}\left(\left(D_{\mathcal{X}}^{2} u\right)^{*}\right)$. In Sect. 4.1 we will give some explicit results for extremal equations involving $\mathcal{P}^{ \pm}$on the Heisenberg group.

\subsection{Fully nonlinear uniformly subelliptic equations}

In this section we consider the general fully nonlinear subelliptic equation

$$
G\left(x, u, D_{\mathcal{X}} u,\left(D_{\mathcal{X}}^{2} u\right)^{*}\right)=0 \text { in } \mathbb{R}^{d}
$$

when $G: \mathbb{R}^{d} \times \mathbb{R} \times \mathbb{R}^{m} \times \mathcal{S}_{m} \rightarrow \mathbb{R}$ satisfies the following form of uniform ellipticity

$$
\mathcal{M}_{\lambda, \Lambda}^{-}(M-N) \leq G(x, r, p, M)-G(x, r, p, N) \leq \mathcal{M}_{\lambda, \Lambda}^{+}(M-N)
$$


for every $(x, r, p) \in \Omega \times \mathbb{R} \times \mathbb{R}^{m}$ and $M, N \in \mathcal{S}_{m}$ with $N \geq 0$. By taking $N=0$ we get

$$
\mathcal{M}_{\lambda, \Lambda}^{-}(M) \leq G(x, r, p, M)-G(x, r, p, 0) \leq \mathcal{M}_{\lambda, \Lambda}^{+}(M),
$$

and, as a consequence, by setting $H(x, r, p):=G(x, r, p, 0)$, one can infer Liouville results for viscosity subsolutions and supersolutions to (29) by comparison with the Eqs. (20) and (22) of the previous section. For this purpose we assume either

$$
G(x, r, p, 0) \geq H_{i}(x, r, p) \quad \forall x \in \mathbb{R}^{d}, r \in \mathbb{R}, p \in \mathbb{R}^{m},
$$

for a concave Hamiltonian of the form (21), or

$$
G(x, r, p, 0) \leq H_{S}(x, r, p)
$$

for a convex $H_{S}$ as in (23).

Corollary 3.12 Assume the vector fields $\mathcal{X}$ are $C^{1,1}$ and satisfy the Hörmander condition (H), (24), (25), and the ellipticity condition (30).

(a) Let (31) hold. Then (LP1) holds for (29) provided that either $u \geq 0$ or $c^{\alpha}(x) \equiv 0$

(b) Let (32) hold. Then, (LP2) holds for (29) provided that either $v \leq 0$ or $c^{\alpha}(x) \equiv 0$.

Proof It is sufficient to observe that $u$ and $v$ satisfy the differential inequalities

$$
\begin{aligned}
& \mathcal{M}_{\lambda, \Lambda}^{-}\left(\left(D_{\mathcal{X}}^{2} u\right)^{*}\right)+H_{i}\left(x, u, D_{\mathcal{X}} u\right) \leq 0 \text { in } \mathbb{R}^{d}, \\
& \mathcal{M}_{\lambda, \Lambda}^{+}\left(\left(D_{\mathcal{X}}^{2} v\right)^{*}\right)+H_{s}\left(x, v, D_{\mathcal{X}} v\right) \geq 0 \text { in } \mathbb{R}^{d},
\end{aligned}
$$

and apply Corollary 3.11.

The same kind of result holds for equations of the form

$$
G\left(x, u, D u,\left(D_{\mathcal{X}}^{2} u\right)^{*}\right)=0 \text { in } \mathbb{R}^{d},
$$

for $G: \mathbb{R}^{d} \times \mathbb{R} \times \mathbb{R}^{d} \times \mathcal{S}_{m} \rightarrow \mathbb{R}$, where the dependence is on the Euclidean gradient $D u$ instead of the horizontal one $D \mathcal{X} u$. We assume $G$ satisfies (30) and either

$$
G(x, r, p, 0) \geq H_{i}(x, r, p), \quad \forall x, p \in \mathbb{R}^{d}, r \in \mathbb{R},
$$

for a concave Hamiltonian of the form (21), but with $b^{\alpha}: \mathbb{R}^{d} \rightarrow \mathbb{R}^{d}$ a vector field in $\mathbb{R}^{d}$, or

$$
G(x, r, p, 0) \leq H_{s}(x, r, p), \quad \forall x, p \in \mathbb{R}^{d}, r \in \mathbb{R},
$$

for a convex Hamiltonian of the form (23) with $b^{\alpha}: \mathbb{R}^{d} \rightarrow \mathbb{R}^{d}$. On $b^{\alpha}$ and $c^{\alpha}$ we make the same assumptions (24), (25), and the fields $\mathcal{X}$ are $C^{1,1}$ and satisfy (H). The arguments leading to Corollaries 3.11 and 3.12 give the following.

Corollary 3.13 Under the conditions listed above a subsolution (resp., supersolution) of (33) with assumption (34) (resp., (35)) verifies the Liouville property (a) (resp., (b)) of Corollary 3.12. 


\subsection{Normalized $p$-Laplacian}

The result of the last section encompasses degenerate equations of the form

$$
-\left|D_{\mathcal{X}} u\right|^{2-p} \operatorname{div} \mathcal{X}\left(\left|D_{\mathcal{X}} u\right|^{p-2} D_{\mathcal{X}} u\right)=0 \text { in } \mathbb{R}^{d},
$$

where $\operatorname{div} \mathcal{X}$ is the intrinsic divergence over the fields of the family $\mathcal{X}$. In fact the operator $E\left(D_{\mathcal{X}} u,\left(D_{\mathcal{X}}^{2} u\right)^{*}\right):=-\left|D_{\mathcal{X}} u\right|^{2-p} \operatorname{div} \mathcal{X}\left(\left|D_{\mathcal{X}} u\right|^{p-2} D_{\mathcal{X}} u\right)$, called normalized or game-theoretic $p$-Laplacian, can be rewritten as $-\operatorname{Tr}\left[A\left(D_{\mathcal{X}} u\right)\left(D_{\mathcal{X}}^{2} u\right)^{*}\right]$, with

$$
A\left(D_{\mathcal{X}} u\right)=I_{m}+(p-2) \frac{D_{\mathcal{X}} u \otimes D_{\mathcal{X}} u}{\left|D_{\mathcal{X}} u\right|^{2}}
$$

In other words,

$$
E\left(D_{\mathcal{X}} u,\left(D_{\mathcal{X}}^{2} u\right)^{*}\right)=-\Delta_{\mathcal{X}} u-(p-2)\left|D_{\mathcal{X}} u\right|^{-2} \Delta_{\mathcal{X}, \infty} u
$$

where $\Delta \mathcal{X}, \infty$ is the $\infty$-Laplacian operator over the fields $\mathcal{X}$. It is immediate to see that

$$
\min \{1, p-1\}|\xi|^{2} \leq A\left(D_{\mathcal{X}} u\right) \xi \cdot \xi \leq \max \{1, p-1\}|\xi|^{2}
$$

showing that $E$ is uniformly subelliptic for $p \in(1, \infty)$. Therefore such nonlinear operators can be compared with Pucci's extremal operators $\mathcal{M}_{\lambda, \Lambda}^{ \pm}$with $\lambda=\min \{1, p-$ $1\}$ and $\Lambda=\max \{1, p-1\}$ over Hörmander vector fields. They were studied recently by several authors, see e.g. [1] for the case of Carnot groups, and the references therein. The game theoretic $p$-Laplace operator on $\mathcal{X}$ is the sublaplacian if $p=2$, whereas for $p=1$ it drives the evolutive equation describing the motion of level sets by sub-Riemannian mean curvature.

\section{The Heisenberg vector fields}

The aim of this section is to specialize the results obtained in the previous one to viscosity subsolutions of (29) fulfilling (30) over Heisenberg vector fields. We briefly recall some standard facts on the Heisenberg group. For further details we refer the reader to the monograph [12]. The Heisenberg group $\mathbb{H}^{d}$ can be identified with $\left(\mathbb{R}^{2 d+1}, \circ\right)$, where $2 d+1$ stands for the topological dimension and the group law $\circ$ is defined by

$$
x \circ y=\left(x_{1}+y_{1}, \ldots, x_{2 d}+y_{2 d}, x_{2 d+1}+y_{2 d+1}+2 \sum_{i=1}^{d}\left(x_{i} y_{i+d}-x_{i+d} y_{i}\right)\right) .
$$

We denote with $x$ a point of $\mathbb{R}^{2 d+1}$ and set

$$
x_{H}:=\left(x_{1}, \ldots, x_{2 d}\right)
$$


The $d$-dimensional Heisenberg algebra is the Lie algebra spanned by the $m=2 d$ vector fields

$$
\begin{aligned}
& X_{i}=\partial_{i}+2 x_{i+d} \partial_{2 d+1}, \\
& X_{i+d}=\partial_{i+d}-2 x_{i} \partial_{2 d+1},
\end{aligned}
$$

for $i=1, \ldots, d$. Such vector fields satisfy the commutation relations

$$
\left[X_{i}, X_{i+d}\right]=-4 \partial_{2 d+1} \text { and }\left[X_{i}, X_{j}\right]=0 \text { for all } j \neq i+d, i \in\{1, \ldots, d\}
$$

and are 1-homogeneous with respect to the family of (anisotropic) dilations

$$
\delta_{\lambda}(x)=\left(\lambda x_{1}, \ldots, \lambda x_{2 d}, \lambda^{2} x_{2 d+1}\right), \lambda>0,
$$

Following [12, Definition 5.1.1], it is useful to consider the following homogeneous norm defined via the stratification property of $\mathbb{H}^{d}$

$$
\rho(x)=\left(\left(\sum_{i=1}^{2 d}\left(x_{i}\right)^{2}\right)^{2}+x_{2 d+1}^{2}\right)^{\frac{1}{4}}
$$

which is 1-homogeneous with respect to the previous group of dilations. We emphasize that this norm is easier to compute than the Carnot-Carathéodory norm.

\subsection{Fully nonlinear PDEs on the Heisenberg group}

In the next result we provide sufficient conditions for the validity of the Liouville property for viscosity subsolutions to (20). We search the Lyapunov functions of property (iii) and (iii') among the radial ones (e.g. $w=\log \rho$ and $W=-\log \rho$ ). Here and in the next examples we exploit a classical chain rule to compute the horizontal gradient and Hessian of a "radial" function with respect to the homogeneous norm $\rho$. Indeed, for a sufficiently smooth radial function $f=f(\rho)$ and given a system of vector fields $\mathcal{X}=\left\{X_{1}, \ldots, X_{m}\right\}$, we have

$$
D_{\mathcal{X}} f(\rho)=f^{\prime}(\rho) D_{\mathcal{X}} \rho
$$

and

$$
D_{\mathcal{X}}^{2} f(\rho)=f^{\prime}(\rho) D_{\mathcal{X}}^{2} \rho+f^{\prime \prime}(\rho) D_{\mathcal{X}} \rho \otimes D_{\mathcal{X}} \rho
$$

In this section we denote the Heisenberg horizontal gradient and symmetrized Hessian by $D_{\mathbb{H}^{d}}$ and $\left(D_{\mathbb{H}^{d}}^{2}\right)^{*}$. We premise the following auxiliary result taken from [21, Lemma 3.1 and Lemma 3.2]. 
Lemma 4.1 Let $\rho$ be defined in (36). Then, for $\left|x_{H}\right| \neq 0$,

$$
D_{\mathbb{H}^{d}} \rho=\frac{\eta}{\rho^{3}}, \quad\left|D_{\mathbb{H}^{d}} \rho\right|^{2}=\frac{\left|x_{H}\right|^{2}}{\rho^{2}} \leq 1,
$$

where $\eta \in \mathbb{R}^{2 d}$ is defined by

$$
\eta_{i}:=x_{i}\left|x_{H}\right|^{2}+x_{i+d} x_{2 d+1}, \quad \eta_{i+d}:=x_{i+d}\left|x_{H}\right|^{2}-x_{i} x_{2 d+1} .
$$

for $i=1, \ldots, d$. Moreover

$$
D_{\mathbb{H}^{d}}^{2} \rho=-\frac{3}{\rho} D_{\mathbb{H}^{d}} \rho \otimes D_{\mathbb{H}^{d}} \rho+\frac{1}{\rho}\left|D_{\mathbb{H}^{d}} \rho\right|^{2} I_{2 d}+\frac{2}{\rho^{3}}\left(\begin{array}{cc}
B & C \\
-C & B
\end{array}\right),
$$

where the matrices $B=\left(b_{i j}\right)$ and $C=\left(c_{i j}\right)$ are defined as follows

$$
b_{i j}:=x_{i} x_{j}+x_{d+i} x_{d+j}, \quad c_{i j}:=x_{i} x_{d+j}-x_{j} x_{d+i}
$$

for $i, j=1, \ldots, d$ (in particular $B=B^{T}$ and $C=-C^{T}$ ). In addition, for a radial function $f=f(\rho)$ we have

$$
\begin{aligned}
D_{\mathbb{H}^{d}}^{2} f(\rho)= & \frac{f^{\prime}(\rho)\left|D_{\mathbb{H}^{d}} \rho\right|^{2}}{\rho} I_{2 d}+2 \frac{f^{\prime}(\rho)}{\rho^{3}}\left(\begin{array}{cc}
B & C \\
-C & B
\end{array}\right) \\
& +\left(f^{\prime \prime}(\rho)-3 \frac{f^{\prime}(\rho)}{\rho}\right) D_{\mathbb{H}^{d}} \rho \otimes D_{\mathbb{H}^{d}} \rho .
\end{aligned}
$$

and its eigenvalues are $f^{\prime \prime}(\rho)\left|D_{\mathbb{H}^{d}} \rho\right|^{2}, 3 f^{\prime}(\rho) \frac{\left|D_{\mathbb{H}^{d}} \rho\right|^{2}}{\rho}$, which are simple, and $f^{\prime}(\rho) \frac{\left|D_{\mathbb{H} d} \rho\right|^{2}}{\rho}$ with multiplicity $2 d-2$.

Theorem 4.2 Let $\mathcal{X}=\left\{X_{1}, \ldots, X_{2 d}\right\}$ be the system of vector fields generating the Heisenberg group $\mathbb{H}^{d}$. Assume that (24) and (25) are in force and

$$
\sup _{\alpha \in A}\left\{b^{\alpha}(x) \cdot \frac{\eta}{\left|x_{H}\right|^{2}}-c^{\alpha}(x) \frac{\rho^{4}}{\left|x_{H}\right|^{2}} \log \rho\right\} \leq \lambda-\Lambda(Q-1)
$$

for $\rho$ sufficiently large and $\left|x_{H}\right| \neq 0$, where $Q=2 d+2$ is the homogeneous dimension of $\mathbb{H}^{d}, b^{\alpha}(x)$ takes values in $\mathbb{R}^{2 d}$, and $\eta=\left(\eta_{i}, \eta_{i+d}\right)$ is defined by (37).

(A) If either $c^{\alpha}(x) \equiv 0$ or $u \geq 0$, then (LP1) for (20) holds with $w=\log \rho$.

(B) If either $c^{\alpha}(x) \equiv 0$ or $v \leq 0$, then (LP2) for (22) holds with $W=-\log \rho$.

Remark 4.3 When $b \equiv c \equiv 0$ and $\lambda=\Lambda=1$ (i.e. (20) becomes $-\Delta_{\mathbb{H} d} u=0$ ) condition (38) gives $\lambda-\Lambda(Q-1)=(2-Q) \geq 0$, which is not satisfied in the Heisenberg group because $Q \geq 4$. This is consistent with the failure of the Liouville property for sub- and supersolutions of the Heisenberg sub-Laplacian that we prove in Sect. 4.2 below. 
Proof We only have to check property (iii) for the Lyapunov function $w(x)=$ $\log \rho(x)$. Note that $\lim _{|x| \rightarrow \infty} w(x)=\infty$ because $\rho \rightarrow \infty$ as $|x| \rightarrow \infty$. By Lemma 4.1 applied to the radial function $w$ the eigenvalues of $\left(D_{\mathbb{H} d}^{2} w\right)^{*}$ are

$$
-\frac{\left|x_{H}\right|^{2}}{\rho^{4}} \text { and } 3 \frac{\left|x_{H}\right|^{2}}{\rho^{4}}, \quad \text { which are simple, }
$$

and

$$
\frac{\left|x_{H}\right|^{2}}{\rho^{4}} \text { with multiplicity } 2 d-2
$$

when $\left|D_{\mathbb{H} d} \rho\right| \neq 0$. Otherwise all the eigenvalues vanish identically and $w$ is trivially a supersolution to (20) because $\mathcal{M}_{\lambda, \Lambda}^{-}\left(\left(D_{\mathbb{H}^{d}}^{2} w\right)^{*}\right)=0$ and $c^{\alpha} u \geq 0$. Hence, we are able to compute the Pucci's minimal operator at points where $\left|x_{H}\right| \neq 0$ owing to Lemma 4.1 as

$$
\mathcal{M}_{\lambda, \Lambda}^{-}\left(\left(D_{\mathbb{H}^{d}}^{2} w\right)^{*}\right)=\{-\Lambda(2 d+1)+\lambda\} \frac{\left|x_{H}\right|^{2}}{\rho^{4}} .
$$

Thus, $w$ is a supersolution at all points where

$$
\{-\Lambda(2 d+1)+\lambda\} \frac{\left|x_{H}\right|^{2}}{\rho^{4}}+\inf _{\alpha \in A}\left\{c^{\alpha}(x) \log \rho-b^{\alpha}(x) \cdot \frac{\eta}{\rho^{4}}\right\} \geq 0,
$$

because $D_{\mathbb{H} d} w=\eta / \rho^{4}$ by Lemma 4.1. In particular, this inequality holds when $\rho$ is sufficiently large under condition (38) by recalling that $Q=2 d+2$. Similarly, one can check that (38) implies that the function $W(\rho)=-\log \rho$ is a subsolution to (22) for $|x|$ sufficiently large at points where $\left|D_{\mathbb{H} d} \rho\right| \neq 0$. Therefore Corollary 3.11 gives the conclusion.

Remark 4.4 Condition (38) is comparable to that obtained in [5, condition (2.17)], but here typical quantities of Carnot groups appear. One may think that the ratio

$$
\frac{\rho^{4}}{\left|x_{H}\right|^{2}}=\frac{\left|x_{H}\right|^{4}+\left|x_{V}\right|^{2}}{\left|x_{H}\right|^{2}}
$$

plays exactly the same role as $|x|^{2}$ in [5, condition (2.17)], while the dimension $d$ of the Euclidean setting is precisely replaced by its sub-Riemannian counterpart $Q$, as expected.

Remark 4.5 A simple condition that implies (38), and therefore the Liouville property, is

$$
\limsup _{|x| \rightarrow \infty} \sup _{\alpha \in A} b^{\alpha}(x) \cdot \frac{\eta}{\left|x_{H}\right|^{2}}<\lambda-\Lambda(Q-1)
$$


since $c \geq 0$. Compare the above condition to that in [5, Remark 2.4]: $Q$ replaces the dimension $d$ of the Euclidean case and $x \in \mathbb{R}^{d}$ is replaced by the vector $\eta /\left|x_{H}\right|^{2} \in \mathbb{R}^{2 d}$, where $\eta$ is defined by (37).

Remark 4.6 Another possible choice of the Lyapunov function $w$ is a power of $\rho$, e.g., $\tilde{w}(\rho)=\frac{\rho^{2}}{2}$. Then we get the Liouville property in the larger class of subsolutions such that

$$
\limsup _{|x| \rightarrow \infty} \frac{u(x)}{\rho(x)^{2}} \leq 0
$$

under the sufficient condition

$$
\sup _{\alpha}\left\{b^{\alpha}(x) \cdot \frac{\eta}{\left|x_{H}\right|^{2}}-c^{\alpha}(x) \frac{\rho^{4}}{\left|x_{H}\right|^{2}}\right\} \leq-\Lambda Q,
$$

which is more restrictive than (38).

We can now give explicit conditions for the Liouville properties for the general subelliptic equation (29) on the Heisenberg group.

Corollary 4.7 Assume that the operator $G$ satisfies (30), $\mathcal{X}=\left\{X_{1}, \ldots, X_{2 d}\right\}$ are the Heisenberg vector fields, and (24), (25), and (38) are satisfied.

(A) Assume (31). Then (LP1) holds for (29) provided that either $u \geq 0$ or $c^{\alpha}(x) \equiv 0$.

(B) Assume (32). Then (LP2) for (29) holds provided that either $v \leq 0$ or $c^{\alpha}(x) \equiv 0$.

Proof It is enough to exploit that $u$ (resp., $v$ ) is a subsolution to (20) (resp., a supersolution to (22)) over the Heisenberg group and then apply Theorem 4.2-(A) (resp., Theorem 4.2-(B)).

We specialize the last corollaries to a class of examples in order to compare with those in [5]. Consider again the fully nonlinear uniformly subelliptic PDE (29) and assume that either

$$
G(x, r, p, 0) \geq-\bar{b}(x) \cdot p-g(x)|p|+\bar{c}(x) r,
$$

or

$$
G(x, r, p, 0) \leq-\bar{b}(x) \cdot p+g(x)|p|+\bar{c}(x) r,
$$

where $\bar{b}: \mathbb{R}^{2 d+1} \rightarrow \mathbb{R}^{2 d}$ and $g: \mathbb{R}^{2 d+1} \rightarrow \mathbb{R}$ are locally Lipschitz, $\bar{c}$ is continuous, $g \geq 0$, and $\bar{c} \geq 0$.

Corollary 4.8 Assume that the operator $G$ in (29) satisfies (30) and

$$
\bar{b}(x) \cdot \frac{\eta}{\left|x_{H}\right|^{2}}+g(x) \frac{|\eta|}{\left|x_{H}\right|^{2}} \leq \bar{c}(x) \frac{\rho^{4}}{\left|x_{H}\right|^{2}} \log \rho+\lambda-\Lambda(Q-1),
$$

for $\rho$ sufficiently large and $\left|x_{H}\right| \neq 0$, where $\eta$ is defined by (37) and $Q=2 d+2$. 
(A) Suppose that (39) holds. Then (LP1) holds for (29) provided that either $c^{\alpha}(x) \equiv 0$ or $u \geq 0$.

(B) Suppose that (40) holds. Then (LP2) holds for (29) provided that either $v \leq 0$ or $c^{\alpha}(x) \equiv 0$

Proof As in the proof of Theorem 4.2 we must only check that $w=\log \rho$ is a supersolution. Observe that $-\left|D_{\mathbb{H}^{d}} w\right|=-\left|\sigma^{T} D w\right|=\min _{|\alpha|=1}\left\{-\alpha \cdot \sigma^{T} D w\right\}$. Hence we can write the right-hand side of the inequality (39) with $p=D_{\mathbb{H}^{d}} w$ as

$$
\inf _{\alpha \in A}\left\{\bar{c} w-(\bar{b}+g \alpha) \cdot \sigma^{T} D w\right\}
$$

where $A=\left\{\alpha \in \mathbb{R}^{2 d}:|\alpha|=1\right\}$. Moreover $D_{\mathbb{H} d} w=\frac{1}{\rho^{4}} \eta$ by Lemma 4.1. Then $w$ is a supersolution where

$$
\{-\Lambda(2 d+1)+\lambda\} \frac{\left|x_{H}\right|^{2}}{\rho^{4}}+\bar{c} \log \rho+\inf _{\alpha \in A}\left\{-(\bar{b}+g \alpha) \cdot \frac{\eta}{\rho^{4}}\right\} \geq 0,
$$

and this inequality is satisfied for $\rho$ large enough if (41) holds.

Arguing in a similar manner we prove (B) by showing that $W=-\log \rho$ is a subsolution.

Example 4.9 (Schrödinger-type equations) For nonnegative subsolutions of the equation

$$
\mathcal{M}_{\lambda, \Lambda}^{-}\left(\left(D_{\mathbb{H} d}^{2} u\right)^{*}\right)+\bar{c}(x) u=0 \text { in } \mathbb{R}^{2 d+1}
$$

the Liouville property holds if

$$
\liminf _{|x| \rightarrow \infty} \bar{c}(x) \frac{\rho^{4}(x)}{\left|x_{H}\right|^{2}} \log \rho(x)>\Lambda(Q-1)-\lambda,
$$

a results that appears to be new even in the linear case $\lambda=\Lambda$. Note that when $\bar{c}(x) \simeq\left|D_{\mathbb{H}^{d}} \rho\right|^{2} \rho^{\gamma}$ at infinity the condition is always satisfied when $\gamma \geq-2$.

Example 4.10 (A horizontal Ornstein-Uhlenbeck equation) Consider subsolutions of the equation

$$
\mathcal{M}_{\lambda, \Lambda}^{-}\left(\left(D_{\mathbb{H}^{d}}^{2} u\right)^{*}\right)-\gamma(x) \eta(x) \cdot D_{\mathbb{H}^{d}} u=0 \text { in } \mathbb{R}^{2 d+1}
$$

where $\gamma(x)>0$ and $\eta$ is defined by (37), i.e.,

$$
\eta(x)=x_{H}\left|x_{H}\right|^{2}+x_{2 d+1} x_{H}^{\perp}, \quad x_{H}^{\perp}:=\left(x_{d+1}, \ldots, x_{2 d},-x_{1}, \ldots,-x_{d}\right) .
$$

Since $\eta \cdot \eta=\left|x_{H}\right|^{2} \rho^{4}$, the condition (41) becomes

$$
\liminf _{|x| \rightarrow \infty} \gamma(x) \rho^{4}(x)>\Lambda(Q-1)-\lambda
$$


and then the Liouville property holds.

We end this subsection with a result on the following equations driven by the Pucci's extremal operators $\mathcal{P}_{\lambda}^{ \pm}$defined by (26) (here $\lambda=\alpha$ )

$$
\begin{array}{ll}
\mathcal{P}_{\lambda}^{-}\left(\left(D_{\mathcal{X}}^{2} u\right)^{*}\right)+H_{i}\left(x, u, D_{\mathcal{X}} u\right)=0 & \text { in } \mathbb{R}^{2 d+1}, \\
\mathcal{P}_{\lambda}^{+}\left(\left(D_{\mathcal{X}}^{2} u\right)^{*}\right)+H_{S}\left(x, u, D_{\mathcal{X}} u\right)=0 & \text { in } \mathbb{R}^{2 d+1} .
\end{array}
$$

Sufficient conditions for the Liouville property can be obtained by comparing $\mathcal{P}^{ \pm}$with $\mathcal{M}^{ \pm}$as follows

$$
\mathcal{P}_{\lambda}^{+}(M) \leq \mathcal{M}_{\lambda, \lambda+(1-d \lambda)}^{+}(M), \quad \mathcal{P}_{\lambda}^{-}(M) \geq \mathcal{M}_{\lambda, \lambda+(1-d \lambda)}^{-}(M), \quad \forall M \in \mathcal{S}_{2 d}
$$

However, by exploiting the representation formulas (27) and (28) for $\mathcal{P}^{ \pm}$we can get optimal sufficient conditions.

Corollary 4.11 Let $\mathcal{X}=\left\{X_{1}, \ldots, X_{2 d}\right\}$ be the system of vector fields generating the Heisenberg group $\mathbb{H}^{d}$. Assume (24), (25), and

$$
\sup _{\alpha \in A}\left\{b^{\alpha}(x) \cdot \frac{\eta}{\left|x_{H}\right|^{2}}-c^{\alpha}(x) \frac{\rho^{4}}{\left|x_{H}\right|^{2}} \log \rho\right\} \leq 4 d \lambda-3
$$

for $\rho$ sufficiently large and $\left|D_{\mathbb{H} d} \rho\right| \neq 0$. Then the same conclusions as in Theorem 4.2 hold for subsolutions of (42) and supersolutions of (43).

Proof The proof is the same as Theorem 4.2 using the Lyapunov function $w(\rho)=\log \rho$ and the formulas (27) and (28). By the expression of the eigenvalues of $\left(D_{\mathbb{H} d}^{2} w\right)^{*}$ in the proof of Theorem 4.2 one finds

$$
\mathcal{P}_{\lambda}^{-}\left(\left(D_{\mathbb{H}^{d}}^{2} w\right)^{*}\right)=(4 d \lambda-3) \frac{\left|x_{H}\right|^{2}}{\rho^{4}} .
$$

Similarly, one uses $W=-\log \rho$ as Lyapunov function for the maximal operator $\mathcal{P}_{\lambda}^{+}$

Remark 4.12 Condition (44) is better than (38) with $\Lambda=\lambda+(1-2 d \lambda)$ and $\lambda<\frac{1}{2 d}$, since

$$
-2 d \lambda-(1-2 d \lambda)(2 d+1)<-3+4 d \lambda .
$$

\subsection{Comparison with the literature and sharpness of the conditions}

In this section we make a comparison with the results in the literature, showing the sharpness of our conditions and those of $[5,20,21]$ via several counterexamples. 


\subsubsection{The Euclidean case}

Corollary 2.4 of [5] states a Liouville-type result that in the case without lower order terms holds for the inequalities

$$
\mathcal{M}_{\lambda, \Lambda}^{-}\left(D^{2} u\right) \leq 0 \text { in } \mathbb{R}^{d}, \quad \mathcal{M}_{\lambda, \Lambda}^{+}\left(D^{2} u\right) \geq 0 \text { in } \mathbb{R}^{d}
$$

the former for viscosity subsolutions bounded above, and the second for supersolutions bounded from below, when $d \leq \frac{\lambda}{\Lambda}+1$. This complements the result of [20] on (5) and (6) recalled in the Introduction, but with a more restrictive condition on $d$, which is, however, still sharp for the Laplacian $(\lambda=\Lambda)$.

The next counterexample shows that the inequalities can have nonconstant solutions when $d>\frac{\lambda}{\Lambda}+1$.

Counterexample 4.13 For $d \geq 2$ set $\beta:=\frac{\Lambda}{\lambda}(d-1)+1$ and consider the function

$$
u_{2}(x)= \begin{cases}\frac{1}{8}\left[\beta(\beta-2)|x|^{4}-2\left(\beta^{2}-4\right)|x|^{2}+\beta(\beta+2)\right] & \text { if }|x|<1 \\ \frac{1}{|x|^{\beta-2}} & \text { if }|x| \geq 1\end{cases}
$$

Since it is radial, the eigenvalues of the Hessian matrix can be computed by [20, Lemma 3.1] and one checks that it is a classical solution to $\mathcal{M}_{\lambda, \Lambda}^{+}\left(D^{2} u_{2}\right) \geq 0$ in $\mathbb{R}^{d}$. Moreover it is bounded and not constant if $\beta>2$, which is equivalent to $d>\lambda / \Lambda+1$, so the Liouville property for supersolutions to $\mathcal{M}^{+}\left(D^{2} u\right)=0$ is false in this case. Similarly, $v_{2}=-u_{2}$ gives a counterexample for solutions to $\mathcal{M}_{\lambda, \Lambda}^{-}\left(D^{2} v_{2}\right) \leq 0$ in $\mathbb{R}^{d}$.

Remark 4.14 The paper [20] studies a similar but different problem with respect to [5], namely, the Liouville property for viscosity supersolutions to $\mathcal{M}_{\lambda, \Lambda}^{-}\left(D^{2} u\right)=0$ in $\mathbb{R}^{d}$ and for subsolutions to $\mathcal{M}_{\lambda, \Lambda}^{+}\left(D^{2} u\right)=0$. They prove it under the less restrictive condition $d \leq \frac{\Lambda}{\lambda}+1$ [20, Theorem 3.2]. Note, however, that their theorem cannot be applied to general uniformly elliptic operators via the inequalities (30), whereas the results in [5] allow such application. The next example shows that also the condition in [20] is optimal.

Counterexample 4.15 (From [20]) Set $\alpha:=\frac{\lambda}{\Lambda}(d-1)+1$ and consider the function

$$
u_{3}(x)= \begin{cases}-\frac{1}{8}\left[\alpha(\alpha-2)|x|^{4}-2\left(\alpha^{2}-4\right)|x|^{2}+\alpha(\alpha+2)\right] & \text { if }|x|<1 \\ -\frac{1}{|x|^{\alpha-2}} & \text { if }|x| \geq 1\end{cases}
$$

which is a classical solution to $\mathcal{M}_{\lambda, \Lambda}^{+}\left(D^{2} u_{3}\right) \leq 0$ in $\mathbb{R}^{d}$. Moreover it is bounded and not constant if $\alpha>2$, which is equivalent to $d>\Lambda / \lambda+1$. Similarly, $v_{3}=-u_{3}$ yields a counterexample for the corresponding property for the minimal operator. 


\subsubsection{The Heisenberg case: sublaplacians}

Liouville's theorem for classical harmonic functions on the Heisenberg group is a consequence of the Harnack inequality, see, e.g., [12, Theorem 8.5.1], or mean-value formulas [17, Theorem 3.1]. However, the Liouville property for classical subsolutions (resp., supersolutions) bounded from above (resp., below) of

$$
-\Delta_{\mathbb{H}} d u=0 \text { in } \mathbb{H}^{d} \simeq \mathbb{R}^{2 d+1}
$$

is false for all dimensions $d$, as the next example shows. We recall that $Q:=2 d+2$ is the homogeneous dimension of $\mathbb{H}^{d}$ and $\rho(x)$ is the homogeneous norm defined in (36).

Counterexample 4.16 The function

$$
\tilde{u}(x)= \begin{cases}\frac{1}{8}\left[Q(Q-2) \rho^{4}-2\left(Q^{2}-4\right) \rho^{2}+Q(Q+2)\right] & \text { if } \rho \leq 1 \\ \frac{1}{\rho^{Q-2}} & \text { if } \rho \geq 1\end{cases}
$$

is a bounded classical supersolution to $-\Delta_{\mathbb{H}^{d}} u=0$ in $\mathbb{R}^{2 d+1}$. Indeed, when $\rho \leq 1$ one applies Lemma 4.1 to the radial function

$$
\tilde{u}=\tilde{f}(\rho)=\frac{1}{8}\left[Q(Q-2) \rho^{4}-2\left(Q^{2}-4\right) \rho^{2}+Q(Q+2)\right]
$$

and gets, at points where $\left|D_{\mathbb{H}^{d}} \rho\right| \neq 0$,

$$
\begin{aligned}
-\Delta_{\mathbb{H} d} \tilde{u}= & -\operatorname{Tr}\left(D_{\mathbb{H}^{d}}^{2} \tilde{f}(\rho)\right) \\
= & -\frac{Q-2}{2 \rho^{2}}\left|x_{H}\right|^{2} \\
& \times\left\{\left[3 Q \rho^{2}-(Q+2)\right]+3\left[Q \rho^{2}-(Q+2)\right]+(2 d-2)\left[Q \rho^{2}-(Q+2)\right]\right\} \\
= & -\frac{Q-2}{2 \rho^{2}}\left|x_{H}\right|^{2} Q\left(\rho^{2}-1\right)(Q+2) \geq 0,
\end{aligned}
$$

due to the fact that $\rho^{2} \leq 1$ and $Q \geq 4$. At points where $\left|x_{H}\right|=0$ all the eigenvalues of $D_{\mathbb{H}^{d}}^{2} \tilde{f}(\rho)$ vanish and hence $\tilde{u}$ is a solution of the sub-Laplace equation. When $\rho \geq 1$, instead, one observes that $\tilde{u}$ is the fundamental solution of the sub-Laplace equations on the Heisenberg group found by Folland [24]. Similarly, $v=-\tilde{u}$ gives a bounded subsolution to $-\Delta_{\mathbb{H} d} u=0$ in $\mathbb{R}^{2 d+1}$.

\subsubsection{The Heisenberg case: fully nonlinear operators}

The Liouville property in this context was first studied by Cutrì and Tchou [21] for viscosity supersolutions bounded from below of $\mathcal{M}_{\lambda, \Lambda}^{-}\left(\left(D_{\mathbb{H} d}^{2} u\right)^{*}\right)=0$ in $\mathbb{R}^{2 d+1}$ and for subsolutions bounded from above to $\mathcal{M}_{\lambda, \Lambda}^{+}\left(\left(D_{\mathbb{H} d}^{2} u\right)^{*}\right)=0$. Their Theorem 5.2 
states that such functions are constant provided that $Q \leq \frac{\Lambda}{\lambda}+1$. The next is a new example showing that this condition is sharp.

Counterexample 4.17 Set $\tilde{\alpha}:=\frac{\lambda}{\Lambda}(Q-1)+1$. We show that for $\tilde{\alpha}>2$, i.e., $Q>\frac{\Lambda}{\lambda}+1$,

$$
u_{4}(x)= \begin{cases}-\frac{1}{8}\left[\tilde{\alpha}(\tilde{\alpha}-2) \rho^{4}-2\left(\tilde{\alpha}^{2}-4\right) \rho^{2}+\tilde{\alpha}(\tilde{\alpha}+2)\right] & \text { if } \rho<1 \\ -\frac{1}{\rho^{\tilde{\alpha}-2}} & \text { if } \rho \geq 1\end{cases}
$$

is a bounded from above classical solution to $\mathcal{M}_{\lambda, \Lambda}^{+}\left(\left(D_{\mathbb{H}^{d}}^{2} u_{4}\right)^{*}\right) \leq 0$ in $\mathbb{R}^{2 d+1}$ and it is not constant. Indeed, denote by $u_{4}(x)=f_{4}(\rho)$. For $\rho<1$ we have

$$
f_{4}^{\prime}(\rho)=-\frac{\tilde{\alpha}-2}{2} \rho\left[\tilde{\alpha} \rho^{2}-(\tilde{\alpha}+2)\right]
$$

and

$$
f_{4}^{\prime \prime}(\rho)=-\frac{\tilde{\alpha}-2}{2}\left[3 \rho^{2} \tilde{\alpha}-(\tilde{\alpha}+2)\right]
$$

Recalling that $\left|D_{\mathbb{H} d} \rho\right|^{2}=\left|x_{H}\right|^{2} / \rho^{2}$, by Lemma 4.1 the eigenvalues of the radial function $f_{4}(\rho)$

$$
\begin{aligned}
& e_{1}=\left|D_{\mathbb{H}^{d}} \rho\right|^{2} f_{4}^{\prime \prime}(\rho)=-\frac{\tilde{\alpha}-2}{2 \rho^{2}}\left|x_{H}\right|^{2}\left[3 \rho^{2} \tilde{\alpha}-(\tilde{\alpha}+2)\right], \\
& e_{2}=3\left|D_{\mathbb{H}^{d}} \rho\right|^{2} \frac{f_{4}^{\prime}(\rho)}{\rho}=-3 \frac{\tilde{\alpha}-2}{2 \rho^{2}}\left|x_{H}\right|^{2}\left[\tilde{\alpha} \rho^{2}-(\tilde{\alpha}+2)\right],
\end{aligned}
$$

which are both simple, and

$$
e_{3}=\left|D_{\mathbb{H}^{d}} \rho\right|^{2} \frac{f_{4}^{\prime}(\rho)}{\rho}=-\frac{\tilde{\alpha}-2}{2 \rho^{2}}\left|x_{H}\right|^{2}\left[\tilde{\alpha} \rho^{2}-(\tilde{\alpha}+2)\right]
$$

which has multiplicity $2 d-2$. Observe that, when $\rho<1$ and $\tilde{\alpha}>2$, the eigenvalues $e_{2}, e_{3}$ are always positive. Moreover, for $\rho^{2} \leq \frac{\tilde{\alpha}+2}{3 \tilde{\alpha}}<1$, also $e_{1}$ is positive and hence $\mathcal{M}_{\lambda, \Lambda}^{+}\left(\left(D_{\mathbb{H} d}^{2} u_{4}\right)^{*}\right) \leq 0$. When $1>\rho^{2}>\frac{\tilde{\alpha}+2}{3 \tilde{\alpha}}, e_{1}<0$, and hence by Lemma 4.1

$$
\begin{aligned}
\mathcal{M}_{\lambda, \Lambda}^{+}\left(\left(D_{\mathbb{H}^{d}}^{2} u_{4}\right)^{*}\right)= & \Lambda \frac{\tilde{\alpha}-2}{2 \rho^{2}}\left|x_{H}\right|^{2}\left[3 \rho^{2} \tilde{\alpha}-(\tilde{\alpha}+2)\right] \\
& +\lambda\left\{\frac{\tilde{\alpha}-2}{2 \rho^{2}}\left|x_{H}\right|^{2}\left[\tilde{\alpha} \rho^{2}-(\tilde{\alpha}+2)\right](2 d-2)\right. \\
& \left.+3 \frac{\tilde{\alpha}-2}{2 \rho^{2}}\left|x_{H}\right|^{2}\left[\tilde{\alpha} \rho^{2}-(\tilde{\alpha}+2)\right]\right\} \\
= & \frac{\tilde{\alpha}-2}{2 \rho^{2}}\left|x_{H}\right|^{2}\left\{\lambda\left[\tilde{\alpha} \rho^{2}-(\tilde{\alpha}+2)\right](2 d-2)+3 \tilde{\alpha} \rho^{2}-3(\tilde{\alpha}+2)\right] \\
& \left.+\Lambda\left[3 \rho^{2} \tilde{\alpha}-(\tilde{\alpha}+2)\right]\right\}
\end{aligned}
$$




$$
\begin{aligned}
& =\frac{\tilde{\alpha}-2}{2 \rho^{2}}\left|x_{H}\right|^{2}\left\{\tilde{\alpha} \rho^{2}[(2 d+1) \lambda+3 \Lambda]-\lambda(2 d+1)(\tilde{\alpha}+2)-\Lambda(\tilde{\alpha}+2)\right\} \\
& =\frac{\tilde{\alpha}-2}{2 \rho^{2}}\left|x_{H}\right|^{2}\left\{\tilde{\alpha} \rho^{2}[(Q-1) \lambda+3 \Lambda]-[\lambda(Q-1)+\Lambda](\tilde{\alpha}+2)\right\} \\
& =\frac{\tilde{\alpha}-2}{2 \rho^{2}}\left|x_{H}\right|^{2}\left\{[\lambda(Q-1)+\Lambda]\left(-\tilde{\alpha}-2+\tilde{\alpha} \rho^{2}\right)+2 \Lambda \tilde{\alpha} \rho^{2}\right\} \\
& \leq \frac{\tilde{\alpha}-2}{2 \rho^{2}}\left|x_{H}\right|^{2}\{-2[\lambda(Q-1)+\Lambda]+2 \Lambda \tilde{\alpha}\} \\
& =\frac{\tilde{\alpha}-2}{2 \rho^{2}}\left|x_{H}\right|^{2}\{-2 \lambda(Q-1)+2 \Lambda(\tilde{\alpha}-1)\}=0,
\end{aligned}
$$

where the last equality is true in view of $\tilde{\alpha}-1=\frac{\lambda}{\Lambda}(Q-1)$. When $\rho>1$ we have

$$
\begin{aligned}
& f_{4}^{\prime}(\rho)=-(2-\tilde{\alpha}) \rho^{1-\tilde{\alpha}}, \\
& f_{4}^{\prime \prime}(\rho)=-(2-\tilde{\alpha})(1-\tilde{\alpha}) \rho^{-\tilde{\alpha}},
\end{aligned}
$$

and the eigenvalues are

$$
\begin{aligned}
& e_{4}=\left|D_{\mathbb{H}^{d}} \rho\right|^{2} f_{4}^{\prime \prime}(\rho)=-\frac{\left|x_{H}\right|^{2}(2-\tilde{\alpha})(1-\tilde{\alpha})}{\rho^{\tilde{\alpha}+2}} \\
& e_{5}=3\left|D_{\mathbb{H}^{d}} \rho\right|^{2} \frac{f_{4}^{\prime}(\rho)}{\rho}=-3 \frac{\left|x_{H}\right|^{2}(2-\tilde{\alpha})}{\rho^{\tilde{\alpha}+2}}
\end{aligned}
$$

and

$$
e_{6}=\left|D_{\mathbb{H}^{d}} \rho\right|^{2} \frac{f_{4}^{\prime}(\rho)}{\rho}=-\frac{\left|x_{H}\right|^{2}(2-\tilde{\alpha})}{\rho^{\tilde{\alpha}+2}}
$$

with multiplicity $2 d-2$. Therefore, for $\rho \geq 1$, we have

$$
\mathcal{M}_{\lambda, \Lambda}^{+}\left(\left(D_{\mathbb{H} d}^{2} u_{4}\right)^{*}\right)=\frac{\left|x_{H}\right|^{2}(2-\tilde{\alpha})}{\rho^{\tilde{\alpha}+2}}[\Lambda(1-\tilde{\alpha})+\lambda(Q-1)]=0 .
$$

Similarly, $v_{4}=-u_{4}$ yields a counterexample for the corresponding property of the minimal operator.

Next we discuss the optimality of our Theorem 4.2 in the case without lower order terms, i.e., $H_{i}=H_{s}=0$. Then the condition (38) becomes $Q \leq \frac{\lambda}{\Lambda}+1$, which is not satisfied in the Heisenberg group because $Q \geq 4$. This is consistent with the failure of the Liouville property for sub- and supersolutions of the Heisenberg Laplacian observed before. The next example shows that the Liouville property fails also for supersolutions bounded from below of $\mathcal{M}_{\lambda, \Lambda}^{+}\left(\left(D_{\mathbb{H} d}^{2} u\right)^{*}\right)=0$ and subsolutions bounded from above of $\mathcal{M}_{\lambda, \Lambda}^{-}\left(\left(D_{\mathbb{H} d}^{2} u\right)^{*}\right)=0$, for all $\lambda, \Lambda$, and $d$. Therefore, we conclude that the presence of suitable lower order terms in Theorem 4.2 is necessary for the Liouville property. 
Counterexample 4.18 Set $\tilde{\beta}:=\frac{\Lambda}{\lambda}(Q-1)+1$. Note that $\tilde{\beta}>2$ because $Q \geq 4>\frac{\lambda}{\Lambda}+1$. In the same way as in Counterexample 4.17, one can verify that the function

$$
u_{5}(x)= \begin{cases}\frac{1}{8}\left[\tilde{\beta}(\tilde{\beta}-2) \rho^{4}-2\left(\tilde{\beta}^{2}-4\right) \rho^{2}+\tilde{\beta}(\tilde{\beta}+2)\right] & \text { if } \rho<1, \\ \frac{1}{\rho^{\tilde{\beta}-2}} & \text { if } \rho \geq 1 .\end{cases}
$$

is a bounded, nonconstant, classical supersolution to $\mathcal{M}_{\lambda, \Lambda}^{+}\left(\left(D_{\mathbb{H} d}^{2} u_{5}\right)^{*}\right)=0$.

\subsection{Equations with Heisenberg Hessian and Euclidean gradient}

Here we consider equations of the form (3), i.e., (33), namely,

$$
G\left(x, u, D u,\left(D_{\mathbb{H}^{d}}^{2} u\right)^{*}\right)=0 \text { in } \mathbb{R}^{d},
$$

with $G: \mathbb{R}^{2 d+1} \times \mathbb{R} \times \mathbb{R}^{2 d+1} \times \mathcal{S}_{2 d} \rightarrow \mathbb{R}$, so they involve the Heisenberg Hessian $D_{\mathbb{H} d}^{2} u$ and the Euclidean gradient $D u$. As at the end of Sect. 3.4 we assume $G$ is uniformly subelliptic and its first order part is bounded from below by a concave Hamiltonian $H_{i}$ or from above by a convex one $H_{s}$. Then Corollary 3.13 gives one of the Liouville properties if we find a suitable super- or subsolution out of a big ball. The next result gives an explicit sufficient condition saying that the vector fields $b^{\alpha}$ in the drift part of $H_{i}$ and $H_{s}$ point toward the origin for $|x|$ large enough, as in the Ornstein-Uhlenbeck operators. It involves the homogeneous norm $\rho$ of the Heisenberg group defined by (36).

Corollary 4.19 Assume that the operator $G$ satisfies (30), where $\mathcal{X}=\left\{X_{1}, \ldots, X_{2 d}\right\}$ are the Heisenberg vector fields, and (24) and (25) hold. Suppose there exist $\gamma_{1}, \ldots, \gamma_{2 d+1} \in \mathbb{R}$ with $\min _{i} \gamma_{i}=\gamma_{o}>0$ and such that

$$
\sup _{\alpha} b^{\alpha}(x) \cdot D \rho(x) \leq-\sum_{i=1}^{2 d+1} \gamma_{i} x_{i} \partial_{i} \rho+o\left(\frac{1}{\rho^{3}}\right) \quad \text { as } \rho \rightarrow \infty .
$$

(A) Assume (34). If either $c^{\alpha}(x) \equiv 0$ or $u \geq 0$, then (LP1) holds for (45).

(B) Assume (35). If either $c^{\alpha}(x) \equiv 0$ or $v \leq 0$, then (LP2) holds for (45).

Proof We check that $w=\log \rho$ is a supersolution. Let $C_{1}:=\Lambda(2 d+1)-\lambda>0$. As in the proof of Theorem 4.2, $w$ is a supersolution at all points where

$$
-C_{1} \frac{\left|x_{H}\right|^{2}}{\rho^{4}}+\inf _{\alpha \in A}\left\{c^{\alpha}(x) \log \rho-b^{\alpha}(x) \cdot \frac{D \rho}{\rho}\right\} \geq 0 .
$$

Since $D \rho=\left(2\left|x_{H}\right|^{2} x_{H}, x_{2 d+1}\right) /\left(2 \rho^{3}\right)$, we get from (46) that the left hand side is larger than

$$
-C_{1} \frac{\left|x_{H}\right|^{2}}{\rho^{4}}+\frac{1}{2 \rho^{4}}\left(2 \sum_{i=1}^{2 d} \gamma_{i} x_{i}^{2}\left|x_{H}\right|^{2}+\gamma_{2 d+1} x_{2 d+1}^{2}+o(1)\right)
$$




$$
\geq \frac{1}{\rho^{4}}\left(\left|x_{H}\right|^{2}\left(\gamma_{o}\left|x_{H}\right|^{2}-C_{1}\right)+\frac{\gamma_{o}}{2} x_{2 d+1}^{2}+o(1)\right) \geq 0 \text {, }
$$

for $\rho$ large enough, by taking either $\left|x_{H}\right|^{2}>C_{1} / \gamma_{o}$, or $\left|x_{H}\right|^{2} \leq C_{1} / \gamma_{o}$ and $x_{2 d+1}^{2}>$ $2 C_{1}^{2} / \gamma_{o}^{2}$.

The last result is based on a condition of positivity of the coefficients $c^{\alpha}$ at infinity similar to Example 4.9.

Corollary 4.20 In the assumptions of Corollary 4.19 replace (46) with

$$
\liminf _{|x| \rightarrow \infty} \inf _{\alpha \in A} c^{\alpha}(x) \log \rho(x)>0,
$$

and either

$$
\limsup _{|x| \rightarrow \infty} \sup _{\alpha \in A} b^{\alpha}(x) \cdot D \rho(x) \leq 0
$$

or

$$
\sup _{\alpha \in A}\left|b^{\alpha}(x)\right|=o(\rho) \quad \text { as } \rho \rightarrow \infty .
$$

Then the conclusions of Corollary 4.19 hold true.

Proof We check again the inequality (47). Condition (49) implies that $-b^{\alpha}(x)$. $D \rho / \rho \geq o(1)$ as $\rho \rightarrow \infty$ uniformly in $\alpha$, and the same occurs under (50) because $D \rho=\bar{O}(1)$. Also $\left|x_{H}\right|^{2} / \rho^{4} \leq 1 / \rho^{2}=o(1)$. Then condition (48) implies (47) for $|x|$ large enough.

Remark 4.21 Corollary 4.19 generalizes to fully nonlinear equations the Liouville properties for linear Ornstein-Uhlenbeck operators with Heisenberg sub-laplacian proved in [33].

The condition (48) in Corollary 4.20 obviously holds if $c^{\alpha}(x) \geq c_{o}>0$ for $|x|$ large enough, and in such case the condition (50) can be weakened to $\sup _{\alpha \in A}\left|b^{\alpha}(x)\right|=$ $o(\rho \log \rho)$.

Funding Open access funding provided by Università degli Studi di Padova within the CRUI-CARE Agreement.

Open Access This article is licensed under a Creative Commons Attribution 4.0 International License, which permits use, sharing, adaptation, distribution and reproduction in any medium or format, as long as you give appropriate credit to the original author(s) and the source, provide a link to the Creative Commons licence, and indicate if changes were made. The images or other third party material in this article are included in the article's Creative Commons licence, unless indicated otherwise in a credit line to the material. If material is not included in the article's Creative Commons licence and your intended use is not permitted by statutory regulation or exceeds the permitted use, you will need to obtain permission directly from the copyright holder. To view a copy of this licence, visit http://creativecommons.org/licenses/by/4.0/.

\section{References}

1. Adamowicz, T., Kijowski, A., Pinamonti, A., Warhurst, B.: Variational approach to the asymptotic mean-value property for the $p$-Laplacian on Carnot groups. Nonlinear Anal. 198, 111893 (2020). 22 
2. Adamowicz, T., Warhurst, B.: Three-spheres theorems for subelliptic quasilinear equations in Carnot groups of Heisenberg-type. Proc. Am. Math. Soc. 144(10), 4291-4302 (2016)

3. Armstrong, S.N., Sirakov, B.: Sharp Liouville results for fully nonlinear equations with power-growth nonlinearities. Ann. Sc. Norm. Super. Pisa Cl. Sci. (5) 10(3), 711-728 (2011)

4. Armstrong, S.N., Sirakov, B., Smart, C.K.: Fundamental solutions of homogeneous fully nonlinear elliptic equations. Commun. Pure Appl. Math. 64(6), 737-777 (2011)

5. Bardi, M., Cesaroni, A.: Liouville properties and critical value of fully nonlinear elliptic operators. J. Differ. Equ. 261(7), 3775-3799 (2016)

6. Bardi, M., Cesaroni, A., Manca, L.: Convergence by viscosity methods in multiscale financial models with stochastic volatility. SIAM J. Financ. Math. 1(1), 230-265 (2010)

7. Bardi, M., Goffi, A.: Liouville results for fully nonlinear equations modeled on Hörmander vector fields: II. Carnot groups and Grushin geometries (forthcoming)

8. Bardi, M., Goffi, A.: New strong maximum and comparison principles for fully nonlinear degenerate elliptic PDEs. Calc. Var. Partial Differ. Equ. 58(6), Art. 184, 20 (2019)

9. Birindelli, I.: Superharmonic functions in the Heisenberg group: estimates and Liouville theorems. NoDEA Nonlinear Differ. Equ. Appl. 10(2), 171-185 (2003)

10. Birindelli, I., Capuzzo Dolcetta, I., Cutrì, A.: Liouville theorems for semilinear equations on the Heisenberg group. Ann. Inst. H. Poincaré Anal. Non Linéaire 14(3), 295-308 (1997)

11. Birindelli, I., Galise, G., Leoni, F.: Liouville theorems for a family of very degenerate elliptic nonlinear operators. Nonlinear Anal. 161, 198-211 (2017)

12. Bonfiglioli, A., Lanconelli, E., Uguzzoni, F.: Stratified Lie groups and potential theory for their subLaplacians. Springer Monographs in Mathematics. Springer, Berlin (2007)

13. Bordoni, S., Filippucci, R., Pucci, P.: Nonlinear elliptic inequalities with gradient terms on the Heisenberg group. Nonlinear Anal. 121, 262-279 (2015)

14. Brandolini, L., Magliaro, M.: Liouville type results and a maximum principle for non-linear differential operators on the Heisenberg group. J. Math. Anal. Appl. 415(2), 686-712 (2014)

15. Caffarelli, L.A., Cabré, X.: Fully Nonlinear Elliptic Equations. American Mathematical Society Colloquium Publications, vol. 43. American Mathematical Society, Providence (1995)

16. Capuzzo Dolcetta, I.: Teoremi di Liouville e stime a priori per equazioni ellittiche semilineari Rend. Sem. Mat. Fis. Milano 68(1998), 1-18 (2001)

17. Capuzzo Dolcetta, I., Cutrì, A.: On the Liouville property for sub-Laplacians. Ann. Scuola Norm. Sup. Pisa Cl. Sci. (4) 25(1997), 239-256 (1998)

18. Capuzzo Dolcetta, I., Cutrì, A.: Hadamard and Liouville type results for fully nonlinear partial differential inequalities. Commun. Contemp. Math. 5(3), 435-448 (2008)

19. Chen, H., Felmer, P.: On Liouville type theorems for fully nonlinear elliptic equations with gradient term. J. Differ. Equ. 255(8), 2167-2195 (2013)

20. Cutrì, A., Leoni, F.: On the Liouville property for fully nonlinear equations. Ann. Inst. H. Poincaré Anal. Non Linéaire 17(2), 219-245 (2000)

21. Cutrì, A., Tchou, N.: Barrier functions for Pucci-Heisenberg operators and applications. Int. J. Dyn. Syst. Differ. Equ. 1(2), 117-131 (2007)

22. Fefferman, C., Phong, D.H.: Subelliptic eigenvalue problems. In: Conference on Harmonic Analysis in honor of Antoni Zygmund, vols. I, II (Chicago, Ill., 1981), Wadsworth Math. Ser., pp. 590-606. Wadsworth, Belmont (1983)

23. Feleqi, E., Rampazzo, F.: Iterated Lie brackets for nonsmooth vector fields. NoDEA Nonlinear Differ. Equ. Appl. 24(6), Art. 61, 43 (2017)

24. Folland, G.B.: A fundamental solution for a subelliptic operator. Bull. Am. Math. Soc. 79, 373-376 (1973)

25. Gidas, B., Spruck, J.: A priori bounds for positive solutions of nonlinear elliptic equations. Commun. Partial Differ. Equ. 6(8), 883-901 (1981)

26. Gilbarg, D., Trudinger, N.S.: Elliptic partial differential equations of second order, Grundlehren der Mathematischen Wissenschaften [Fundamental Principles of Mathematical Sciences], vol. 224, 2nd edn. Springer, Berlin (1983)

27. Goffi, A.: Some new Liouville-type results for fully nonlinear PDEs on the Heisenberg group. Nonlinear Anal. 200, 112013 (2020). 18

28. Grigor'yan, A.: Analytic and geometric background of recurrence and non-explosion of the Brownian motion on Riemannian manifolds. Bull. Am. Math. Soc. (N.S.) 36(2), 135-249 (1999) 
29. Kogoj, A.E., Lanconelli, E.: Liouville theorem for $X$-elliptic operators. Nonlinear Anal. 70(8), 29742985 (2009)

30. Kogoj, A.E., Lanconelli, E.: $L^{p}$-Liouville theorems for invariant partial differential operators in $\mathbb{R}^{n}$. Nonlinear Anal. 121, 188-205 (2015)

31. Leoni, F.: Explicit subsolutions and a Liouville theorem for fully nonlinear uniformly elliptic inequalities in halfspaces. J. Math. Pures Appl. (9) 98(5), 574-590 (2012)

32. Li, Y.Y., Nguyen, L., Wang, B.: Towards a Liouville theorem for continuous viscosity solutions to fully nonlinear elliptic equations in conformal geometry. In: Geometric Analysis, Progress in Mathematics, vol. 333, pp. 221-244. Birkhäuser (2020)

33. Mannucci, P., Marchi, C., Tchou, N.: The ergodic problem for some subelliptic operators with unbounded coefficients. NoDEA Nonlinear Differ. Equ. Appl. 23(4), Art. 47, 26 (2016)

34. Mannucci, P., Marchi, C., Tchou, N.: Singular perturbations for a subelliptic operator. ESAIM Control Optim. Calc. Var. 24(4), 1429-1451 (2018)

35. Nadirashvili, N., Tkachev, V., Vlăduţ, S.: Nonlinear Elliptic Equations and Nonassociative Algebras. Mathematical Surveys and Monographs, vol. 200. American Mathematical Society, Providence (2014)

36. Porretta, A., Priola, E.: Global Lipschitz regularizing effects for linear and nonlinear parabolic equations. J. Math. Pures Appl. (9) 100(5), 633-686 (2013)

37. Priola, E., Zabczyk, J.: Liouville theorems for non-local operators. J. Funct. Anal. 216(2), 455-490 (2004)

38. Protter, M.H., Weinberger, H.F.: Maximum Principles in Differential Equations. Springer, New York (1984). Corrected reprint of the 1967 original

39. Pucci, C.: Operatori ellittici estremanti. Ann. Mat. Pura Appl. 4(72), 141-170 (1966)

40. Quittner, P., Souplet, P.: Superlinear Parabolic Problems. Blow-up, Global Existence and Steady States, 2nd edn. Birkhäuser/Springer, Cham (2019)

41. Rossi, L.: Non-existence of positive solutions of fully nonlinear elliptic equations in unbounded domains. Commun. Pure Appl. Anal. 7(1), 125-141 (2008)

Publisher's Note Springer Nature remains neutral with regard to jurisdictional claims in published maps and institutional affiliations. 